\title{
Mutant generation by allelic exchange and genome resequencing of the biobutanol organism Clostridium acetobutylicum ATCC 824
}

\author{
Muhammad Ehsaan', Wouter Kuit ${ }^{1,2}$, Ying Zhang ${ }^{1}$, Stephen T. Cartman ${ }^{1,4}$, John T. Heap ${ }^{1,3}$, Klaus Winzer ${ }^{1}$
} and Nigel P. Minton ${ }^{1 *}$

\begin{abstract}
Background: Clostridium acetobutylicum represents a paradigm chassis for the industrial production of the biofuel biobutanol and a focus for metabolic engineering. We have previously developed procedures for the creation of inframe, marker-less deletion mutants in the pathogen Clostridium difficile based on the use of pyrE and codA genes as counter selection markers. In the current study we sought to test their suitability for use in C. acetobutylicum.

Results: Both systems readily allowed the isolation of in-frame deletions of the C. acetobutylicum ATCC 824 spo0A and the cac824/genes, leading to a sporulation minus phenotype and improved transformation, respectively. The pyrE-based system was additionally used to inactivate a putative glycogen synthase (CA_C2239, glgA) and the pSOL1 amylase gene (CA_P0168, amyP), leading to lack of production of granulose and amylase, respectively. Their isolation provided the opportunity to make use of one of the key pyrE system advantages, the ability to rapidly complement mutations at appropriate gene dosages in the genome. In both cases, their phenotypes were restored in terms of production of granulose ( $g / g A)$ and amylase (amyP). Genome re-sequencing of the ATCC 824 COSMIC consortium laboratory strain used revealed the presence of 177 SNVs and 49 Indels, including a 4916-bp deletion in the pSOL1 megaplasmid. A total of 175 SNVs and 48 Indels were subsequently shown to be present in an 824 strain re-acquired (Nov 2011) from the ATCC and are, therefore, most likely errors in the published genome sequence, NC_003030 (chromosome) and NC_001988 (pSOL1).

Conclusions: The codA or pyrE counter selection markers appear equally effective in isolating deletion mutants, but there is considerable merit in using a pyrE mutant as the host as, through the use of ACE (Allele-Coupled Exchange) vectors, mutants created (by whatever means) can be rapidly complemented concomitant with restoration of the pyrE allele. This avoids the phenotypic effects frequently observed with high copy number plasmids and dispenses with the need to add antibiotic to ensure plasmid retention. Our study also revealed a surprising number of errors in the ATCC 824 genome sequence, while at the same time emphasising the need to re-sequence commonly used laboratory strains.
\end{abstract}

Keywords: Allelic exchange, In-frame deletion, Counter selection marker, codA, pyrE, Clostridium acetobutylicum, Whole genome re-sequencing

\footnotetext{
*Correspondence: Nigel.Minton@nottingham.ac.uk

${ }^{1}$ Clostridia Research Group, BBSRC/EPSRC Synthetic Biology

Research Centre (SBRC), University of Nottingham, University Park,

Nottingham NG7 2RD, UK

Full list of author information is available at the end of the article
} 


\section{Background}

Clostridium species are a diverse and large grouping of anaerobic, endospore-forming bacteria of importance both to medicine and industry [1]. To many, the term 'clostridia' is synonymous with disease, a consequence of the exploits of such notorious human and animal pathogens as Clostridium botulinum, Clostridium tetani, Clostridium perfringens and Clostridium difficile [2]. The vast majority of the genus are, however, entirely benign and produce all manner of useful chemicals and fuels [3]. Of particular note are the saccharolytic species able to produce the biofuel $n$-butanol typified by Clostridium beijerinckii, Clostridium saccharolyticum, Clostridium saccharoperbutylacetonicum and Clostridium acetobutylicum [4]. All four species are associated with the Acetone-Butanol-Ethanol (ABE) commercial process [5], but it is the latter species that garners the most attention. Accordingly, attempts to develop tools for manipulating metabolism at the genetic level, both to understand and bring about improvements to the fermentative process, have tended to be skewed towards Clostridium acetobutylicum [6].

Early strategies that sought to use recombinationbased methods to inactivate genes of $C$. acetobutylicum were handicapped by the lack of counter-selection markers for use in clostridia and, therefore, merely relied on the integration of the entire plasmid carrying an internal segment of the targeted gene [7-10]. As the plasmid carried a marker gene coding for resistance to an antibiotic, the desired single crossover integrant could be selected on the basis of acquisition of resistance to that antibiotic through its inclusion in the agar medium. Such mutants were intrinsically unstable, a consequence of the presence of duplicated DNA regions via which homologous recombination led to excision of the integrated mutagenic plasmid. Subsequently, researchers turned to the use of group II intron retargeting systems in which an intron-encoding segment of DNA could be deliberately inserted into the genomic target from where it could not excise [11-13]. The mutants generated were, therefore, extremely stable. Clones carrying the inserted intronencoding DNA could be detected by appropriate PCR screening, or in the case of the ClosTron, through selection for acquisition of resistance to erythromycin due to the incorporation of an inactivated ermB gene, or RAM (Retrotransposition Activated Marker), that became functional as a consequence of the successful insertion of the group II intron-encoding DNA into the genomic target [14-16].

The efficiency, reproducibility, speed and ease of use have led ClosTron technology to become the most widely used clostridial mutagen [17]. As with any insertional tool, however, its use is disadvantaged by the possible occurrence of polar effects on downstream genes which can complicate the subsequent analysis of mutant phenotypes. As a consequence, marker-less, in-frame deletions generated by homologous recombination are preferred. Accordingly, in the past few years a number of groups have developed such methods through the successful deployment of a range of counter-selection markers first exemplified in other microbial species. A number of the markers first reported could not be used in a standard wild-type host, but required a specific mutant background. These included the genes galK (galactokinase), pyrF (5-phosphate decarboxylase) and upp (uracil phosphor-ribosyltransferase), exemplified in C. perfringens [18], in Clostridium thermocellum [19] and in C. acetobutylicum [20], respectively. Thereafter, a number of alternative counter selection markers have been described that can be used in a wild-type background, most notably use of the E. coli genes $\operatorname{codA}$ (cytosine deaminase) and $m a z F$ (mRNA interferase) in C. difficile [21] and $C$. acetobutylicum [22], respectively, and the exploitation of the Thermoanaerobacterium saccharolyticum tdk (thymidylate synthetase) and C. thermocellum hpt (hypoxanthine phosphoribosyl transferase) genes to make knock-outs in C. thermocellum [23].

Of particular significance was the development of a method, now termed Allele-Coupled Exchange (ACE), which allows the rapid insertion of heterologous DNA, without inherent limits on size or complexity, into the genome [24]. Following integration of the plasmid by single-crossover recombination, the system is designed such that during the desired second recombination event, a plasmid borne allele becomes 'coupled' to a genome located allele which leads to the creation of a new selectable allele, allowing the isolation of double-crossover cells. The use of highly asymmetric homology arms dictates the order of recombination events. A long homology arm directs the first recombination event (plasmid integration) and a much shorter homology arm directs the second recombination event (plasmid excision). Whilst a number of different genetic loci may be used to insert heterologous DNA via ACE, one exemplification of the method exploits the native pyrE gene, bringing about its inactivation by replacement of the wild-type allele with a mutant allele lacking approximately $300 \mathrm{bp}$ from the $3^{\prime}$ end of the structural gene. The $p y r E$ gene encodes orotate phosphoribosyltransferase [E.C.2.4.2.10] which, in common with PyrF, is an enzyme involved in de novo pyrimidine biosynthesis. It may be used as a positive/ negative selection marker as it is essential in the absence of exogenous pyrimidines and renders 5-fluoroorotate (FOA) toxic to cells. It follows that a heterologous $p y r E$ gene can be used as a counter-selection marker in such a strain, in an equivalent manner to $p y r F$ [19], a facility 
that was demonstrated in two different strains of $C$. difficile using a heterologous pyrE allele from Clostridium sporogenes [25]. Crucially, however, the design of the created pyrE mutant strain is such that its $p y r E$ allele can be rapidly (2 days) restored to wild-type using an appropriate ACE correction vector allowing the specific in-frame deletion mutant to be characterised in a clean, wild-type background. Moreover, this facility provides the parallel opportunity to complement the mutant at an appropriate gene dosage, through insertion of a wild-type copy of the gene, either under the control of its native promoter or the strong $\mathrm{P}_{\mathrm{fdx}}$ promoter (derived from the ferredoxin gene of Clostridium sporogenes), concomitant with restoration of the pyrE allele back to wild-type [25].

In the present study we have extended the significant advantages of the pyrE-based system to $C$. acetobutylicum through appropriate deployment of ACE. At the same time, we took the opportunity to test the suitability of using $\operatorname{cod} A$ as a counter selection marker. During the course of this study, genome sequencing of the strains used revealed the presence of a significant number of SNVs (Single Nucleotide Variations) and Indels (Insertions and Deletions) compared to that expected, emphasising the need for researchers to both establish the sequence of the strains in use in their laboratory and to appropriately curate their laboratory strains.

\section{Results}

\section{Establishment of pyrE and codA Knock-out Vectors for use in C. acetobutylicum}

The use of pyrE as a counter selection marker in C. acetobutylicum required the construction of a knock-out (KO) vector suited to this clostridial species and the availability of an appropriate pyrE mutant host. The required $p y r E$ (CA_C0027) mutant (CRG1545), lacking approximately $300 \mathrm{bp}$ from the $3^{\prime}$-end of the gene, has been previously made using ACE [24]. The existing pyrE-based, KO plasmid, pMTL-YN3 [25], used for making allelic exchange mutants in C. difficile 630 is based on a plasmid replicon (that of pCB102 [26]) shown to be replication defective in this particular clostridial strain [25]. Plasmid pMTLYN3 additionally carries a functional copy of the pyrE gene of C. sporogenes ATCC 15579 that had been transcriptionally fused to a $C$. perfringens catP gene [27]. As the pIM13 replicon has previously proven most useful for allelic exchange vectors in C. acetobutylicum [24], the pCB102-derived [26] replicon of plasmid pMTLYN3 was replaced with that of pIM13 (Methods) and the plasmid obtained designated pMTL-ME3 (Fig. 1). To test that the C. sporogenes ATCC 15579 was functional in C. acetobutylicum, CRG1545 was transformed either with plasmid pMTL-ME3 or a vector control lacking pyrE, pMTL85141. Transformants were selected on
CGM supplemented with $15 \mu \mathrm{g} / \mathrm{ml}$ thiamphenicol (Tm) and $20 \mu \mathrm{g} / \mathrm{ml}$ uracil and incubated for $24 \mathrm{~h}$. Single colonies were re-streaked onto the same medium followed by streaking onto CBM agar, without uracil. All the cells transformed with pMTL-ME3 showed growth similar to that of the C. acetobutylicum ATCC 824 wild-type but the cells transformed with the control vector pMTL85141 and the plasmid-free CRG1545 grew very poorly on minimal medium (Additional file 4: Fig. S1). These data confirmed that the C. sporogenes ATCC 1557 pyrE gene was functional in C. acetobutylicum ATCC 824 and could most likely be used as a counter selection marker.

Similar to pMTL-YN3, the previously constructed $\operatorname{codA}$-based KO vector pMTL-SC7315 used in C. difficile [21] is based on the replicon of pCB102 [26]. Accordingly, its replication region was also changed to that of pIM13 (Methods) to give the plasmid pMTL-SC7515 (Fig. 1). Cytosine deaminase (EC 3.5.4.1) converts the innocuous pyrimidine analogue 5-fluorocytosine (FC) into the highly toxic 5-fluorouracil (FU). FU toxicity occurs via uracil phosphoribosyltransferase (EC 2.4.2.9), followed by a series of steps that result in irreversible inhibition of thymidylate synthase, a key enzyme in nucleotide biosynthesis, and misincorporation of fluorinated nucleotides into DNA and RNA $[28,29]$. As the genome of $C$. acetobutylicum ATCC 824 [30] lacks any obvious codA gene but does carry a homologue of upp (CA_C2879), it would be predicted that the introduction of a functional $\operatorname{cod} A$ gene should result in heightened sensitivity to FC. To test this assumption, the ATCC 824 wild-type was independently transformed with the $\operatorname{cod} A$-based plasmid pMTL-SC7515 and the vector PMTL85151 [31] and the sensitivity of the resultant transformants to FC assessed. The MIC of FC on CBM was reduced from $1 \mathrm{mg} / \mathrm{ml}$ (for the wild-type and pMTL85151 empty-vector control strain) to $100 \mu \mathrm{g} / \mathrm{ml}$ in those cells carrying pMTLSC7515 (data not shown). This confirmed that $\operatorname{codA}$ could most likely form the basis of a counter selection marker in C. acetobutylicum.

\section{Establishment of ACE pyrE correction, complementation and overexpression vectors}

A set of three ACE vectors were previously [24] established for use in C. difficile knock-out mutants that provide the facility to either (1) simply 'correct' the pyrE allele of the host back to wild-type (a so-called pyrE 'correction' vector), thereby allowing the establishment of a $\mathrm{KO}$ phenotype of a gene of interest in an otherwise wild-type background; (2) provide cloning sites downstream of the pyrE allele that allow a functional copy of the $\mathrm{KO}$ gene, together with its own promoter, to be inserted into the genome concomitant with correction of the pyrE allele, thereby allowing complementation at an 


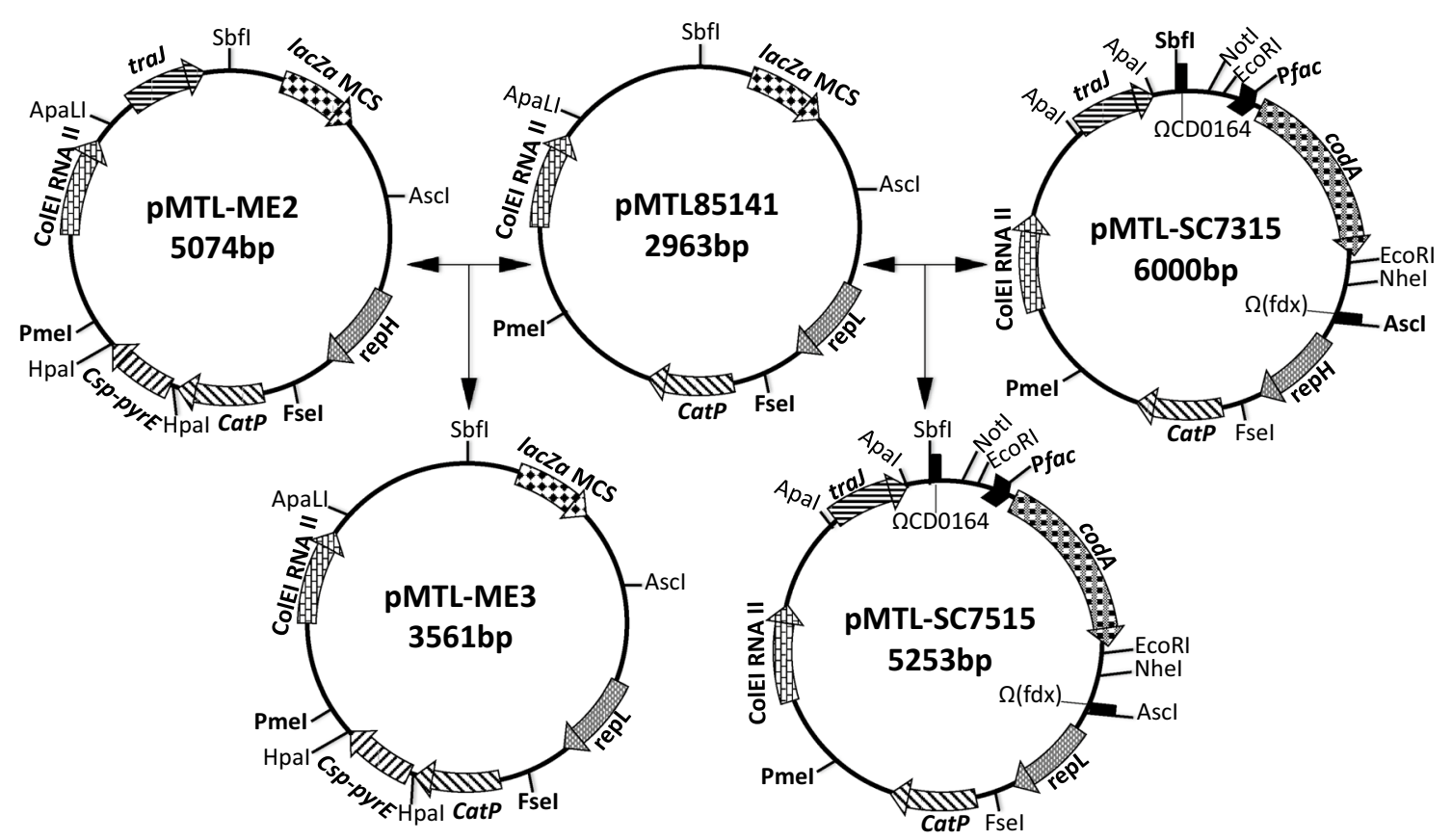

Fig. 1 Clostridial KO vector PMTL-ME3 and PMTL-SC7515. Plasmid components are: CatP, the catP gene of C. perfringens conferring thiamphenicol resistance; Csp-pyrE, the pyrE gene of C. sporogenes; ColE1 RNAll, the replication region of the E.coli plasmid ColE1; traJ, transfer function of plasmid RP4 oriT region; $\Omega C D 0164$, a transcriptional terminator isolated from downstream of the C. difficile strain 630 CD0164 gene; lacZa MCS, the lacZ gene encoding the alpha fragment of the E.coli $\beta$-galactosidase, containing a multiple cloning site, MCS, region derived from plasmid pMTL20; $\Omega(\mathrm{fdx})$, a transcriptional terminator of the ferredoxin gene of C. pasteurianum; repL is the replication protein of plasmid plM13; codA, the cytosine deaminase gene of $E$. coli, and; the promoter in front of $\operatorname{codA}$ is the $\mathrm{P}_{\mathrm{fac}}$ promoter of pMTL007

appropriate gene dosage and (3) provide cloning sites that are preceded by the strong $\mathrm{P}_{f d x}$ promoter that facilitate overexpression of the complementing gene. In C. difficile 630, these vectors were designated pMTL-YN1, pMTLYN1C and pMTL-YN1X, respectively [23]. Accordingly, equivalent vectors were made for $C$. acetobutylicum, namely pMTL-ME6, pMTL-ME6C and pMTL-ME6X (Methods). As a quick check to establish that each vector was capable of correcting the pyrE allele, all three were independently transformed into CRG1545 and plated on CGM medium containing $20 \mu \mathrm{g} / \mathrm{ml}$ uracil and $15 \mu \mathrm{g} / \mathrm{ml}$ $\mathrm{Tm}$, and selected colonies restreaked onto CBM lacking uracil and any antibiotic supplementation. In all cases, prototrophic colonies were isolated consistent with the correction of the pyrE allele.

\section{Creation of mutants using $\operatorname{cod} A$ as negative selection marker}

To demonstrate the utility of $\operatorname{cod} A$ as a counter selection marker in the generation of $\mathrm{KO}$ mutants, two genes were targeted, namely spoOA and the cac824I encoding the principal type II restriction system of $C$. acetobutylicum. Appropriate $\mathrm{KO}$ cassettes, essentially comprising approximately $750 \mathrm{bp}$ from upstream and downstream of the target gene, were assembled and cloned into pMTL-SC7515 as described in "Methods". The two plasmids generated (pMTL-SC7515::spoOA and pMTLSC7515::cac824I) were transformed into the wild-type strain and the procedure described in "Methods" implemented. This involved initially purifying single crossover integrants (through the selection of large, faster-growing transformants), transferring to un-supplemented CGM medium for 2-3 days to allow cells to undergo a second recombination event and then selecting FC-resistant cells

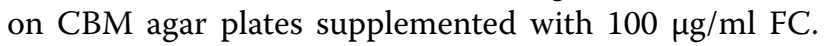
All of those cells that retain the $\operatorname{cod} A$ gene (either those still containing the autonomously replicating plasmid, or those in which the plasmid had integrated) are sensitive to FC and are, therefore, unable to grow. In contrast, those cells in which the plasmid has excised, through a second recombination event, and has been lost due to segregational instability, are able to grow in the presence of FC. The loss of the KO vector was confirmed by replica plating on CGM with or without Tm supplementation.

In the case of the experiment that targeted spoOA, of the four random, $\mathrm{FC}^{\mathrm{R}}$ colonies screened by PCR using primers flanking the gene (Cac-spo0A-sF2 and Cacspo0A-sR2), one generated a DNA fragment of a size 
(2114 bp) equivalent to the wild-type, the other three generated a 1679-bp DNA fragment consistent with the presence of the desired deletion (Fig. 2a). Nucleotide sequencing of the later fragments confirmed the presence of the expected in-frame deletion. Consistent with a sporulation defect, all three putative mutants were shown

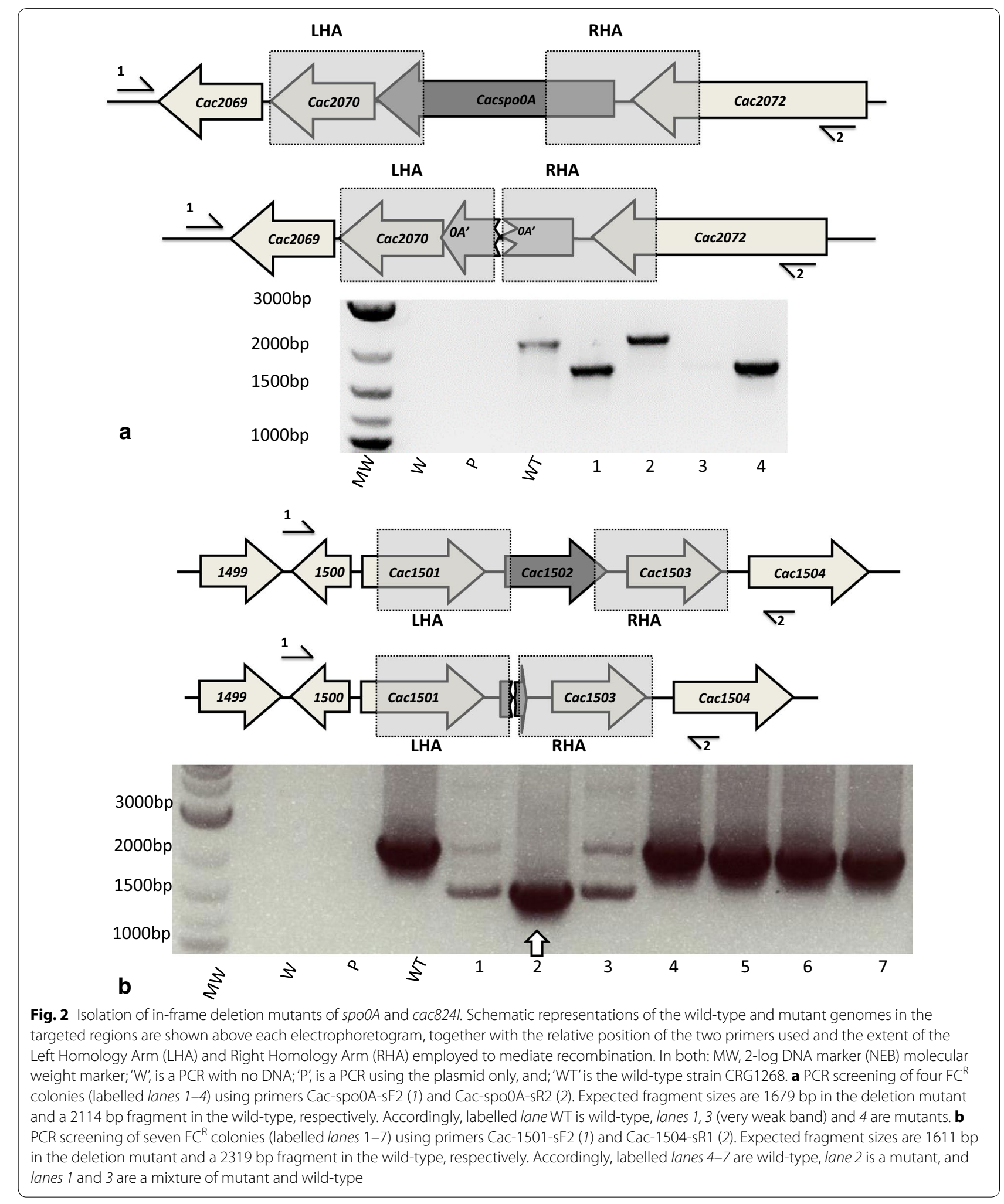


by microscopy to lack phase-bright spores and were unable to form colonies after being subjected to heat treatment at $80{ }^{\circ} \mathrm{C}$ for $10 \mathrm{~min}$ and plating on CGM agar and incubation for $24 \mathrm{~h}$ (data not shown).

In the experiment designed to $\mathrm{KO}$ cac824I, of the seven $\mathrm{FC}^{\mathrm{R}}$ colonies screened only one of them (clone 2) was confirmed as an in-frame deletion mutant, by generation of an appropriately sized fragment with the correct sequence (Fig. 2b). Four of the clones (clones 4-7) appeared to have reverted back to wild-type whereas 1 and 3 appeared to be composed of a mixture of both wild-type and the in-frame deletion mutant. Insertional inactivation or deletion of cac824I by other groups has led to the observation that mutant cells can be transformed with unmethylated DNA as efficiently as with methylated plasmid DNA [32, 33]. Consistent with this finding, $1.5 \times 10^{4}$ transformants per $\mu$ g DNA were obtained when $\Delta c a c 824 I$ was transformed with unmethylated plasmid compared to wild-type where no colonies were obtained.

\section{In-frame deletion of spoOA and its complementation using pyrE correction vectors}

In parallel to the experiments with the $\operatorname{cod} A$-based vector pMTL-SC7515, the equivalent spo0A KO cassette was cloned into the pyrE-based KO vector pMTL-ME3, transformed into the pyrE mutant $C$. acetobutylicum strain CRG1545 and subjected to the mutant isolation procedure outlined in "Methods". After two passages of nine independent $\mathrm{Tm}^{\mathrm{R}}$ transformants on CGM supplemented with $15 \mu \mathrm{g} / \mathrm{ml} \mathrm{Tm}$, three of the visible larger colonies were single crossover integrants (two RH, one LH). Single crossover integrants were streaked onto CGM medium supplemented with $20 \mu \mathrm{g} / \mathrm{ml}$ uracil and incubated for 2-3 days to allow cells to undergo a second recombination event. Bacterial cell suspensions in PBS were spread on CGM supplemented with $400 \mu \mathrm{g} / \mathrm{ml} \mathrm{FOA}$ and $1 \mu \mathrm{g} / \mathrm{ml}$ uracil. The loss of the vector in those cells that grew was confirmed by demonstration that they could no longer grow when patch plated onto CGM medium containing $15 \mu \mathrm{g} / \mathrm{ml} \mathrm{Tm}$. A total of nine randomly selected $\mathrm{Tm}^{\mathrm{S}}$ clones were screened using spoOA flanking PCR primers Cac-spo0A-sF2 and Cac-spo0A-sR2. A DNA fragment of a size (2114 bp) equivalent to the wild-type was obtained with one clone; five clones generated a 1679-bp fragment consistent with the presence of the desired deletion while the remaining three generated both a wild-type and mutant DNA fragment (Fig. 3a). Nucleotide sequencing of the 1679-bp fragment of the five mutants confirmed the presence of the in-frame deletion.

Prior to measurements of their phenotype, the pyrE allele of all the mutants was corrected back to wild-type using the ACE correction vector pMTL-ME6 (Fig. 3b).
Their sporulation minus phenotype was confirmed by the demonstration that none formed phase-bright spores after 2 to 5 days growth in CBM supplemented with $\mathrm{CaCO}_{3}$ and that they could no longer form CFU when plated on CGM after an $80{ }^{\circ} \mathrm{C}$ heat shock for $10 \mathrm{~min}$ (Fig. 4). In parallel to correction of the pyrE locus, complementation and overexpression experiments were undertaken with one of the mutants, CRG3520. Accordingly, the spoOA gene together with its own promoter was cloned into pMTL-ME6C (yielding the pMTL-ME6C::spo0A) and into plasmid pMTL-ME6X downstream of the strong $\mathrm{P}_{f d x}$ promoter [14], to give plasmid pMTL-ME6X::spoOA. The two plasmids were transformed into CRG3520, selecting for $\mathrm{Tm}^{\mathrm{R}}$, and then restreaked onto CBM media lacking uracil to select for clones in which the pyrE gene had been restored to wild-type. Confirmation that in both instances the spoOA gene had become integrated downstream of repaired $p y r E$ gene was obtained using the flanking primers Cac0026-sF2 and Cac-hydA-sR2 (Fig. 3c, d). Those mutant cells in which spoOA was inserted downstream of the corrected pyrE locus under the transcriptional control of its native promoter or the $\mathrm{P}_{f d x}$ promoter were completely restored to a wild-type phenotype, in terms of the presence of phase-bright spores in mature cultures and CFU after heat shock. An estimate of the number of spores produced by the various cultures is shown in Fig. 4.

Although overexpression of spoOA using $\mathrm{P}_{f d x}$ promoter did not show any change in the sporulation frequency, a clear phenotypic change was observed as colonies of the overexpressed strain were visibly bigger compared to complemented and wild-type strains when grown on CBM medium after $24 \mathrm{~h}$ (Additional file 4: Fig. S2).

\section{In-frame deletion of the a-amylase gene and its complementation using pyrE}

To further test the system, and exemplify the utility of overexpressing complementing genes at the pyrE locus in mutant strains, it was desirable to use a mutant which had an easily visualised phenotype. An ideal candidate would be a secreted enzyme for which there is a simple plate-based assay that could measure zones of hydrolysis around a colony. Production of pectinase was initially considered to be an ideal candidate. To rapidly identify such a gene, intron insertion mutants were made using ClosTron technology [14] in CA_C0355, annotated as encoding polygalacturonase/pectinase, at two distinct insertion sites, 656a and $1167 \mathrm{~s}$. No difference was observed in the size of the halo formation in the mutants compared to wild-type after staining of plates with iodine [34] (data not shown). This indicated either the targeted gene was not responsible for the observed pectinase 


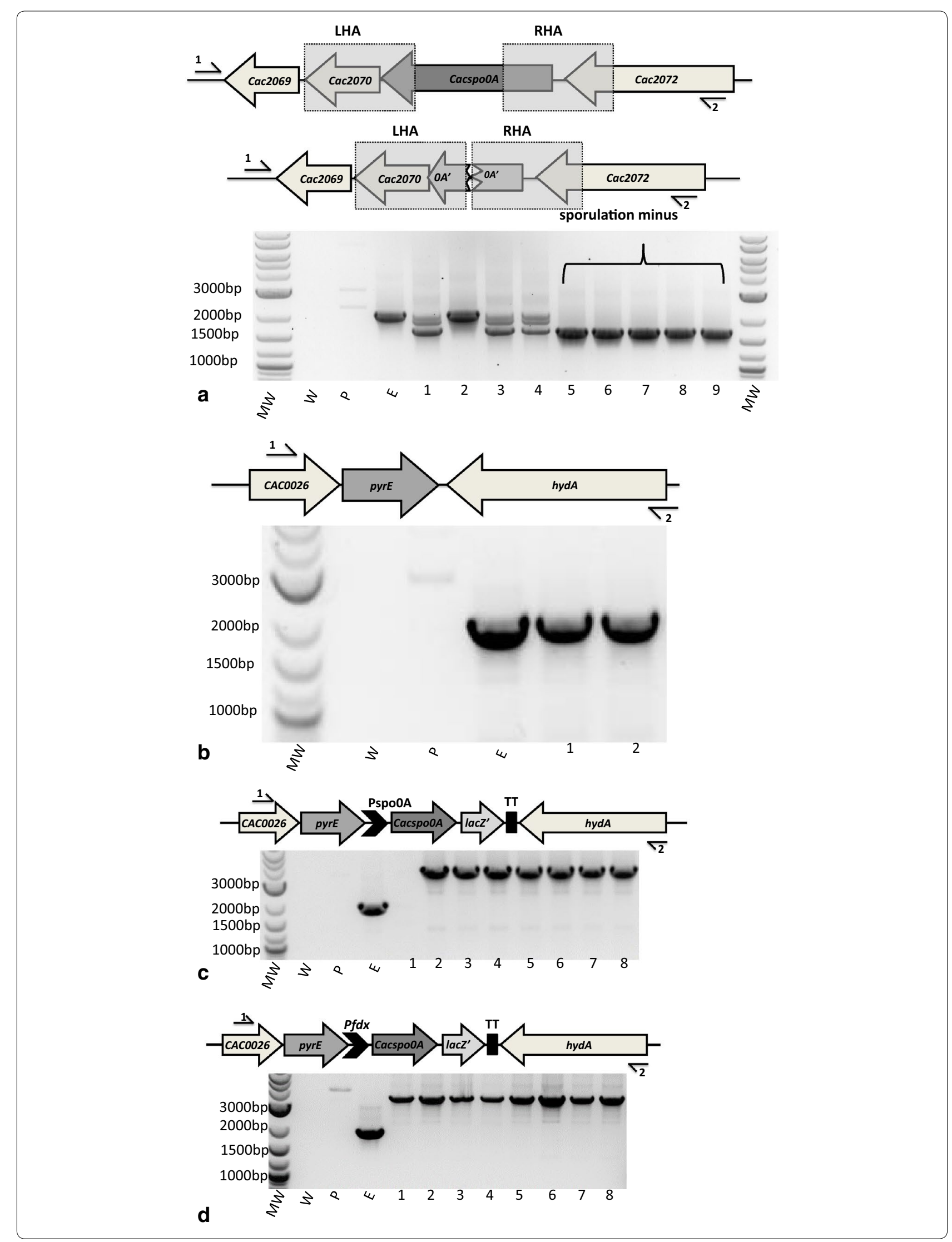


(See figure on previous page.)

Fig. 3 Screening of SpoOA mutants and ACE corrected/complemented/overexpressed clones. Key MW, 2-log DNA marker (NEB) molecular weight marker; 'W', is a PCR with no DNA; 'P', is a PCR using the plasmid only, and;' 'E' is the pyrE minus parent strain CRG1545. On b, c the final arrangement of the chromosomal region in the pyrE repaired strains is illustrated. Components are: $d c d$, deoxycytidine triphosphate deaminase; cac0026, hypothetical protein; pyrE, orotate phosphoribosyltranspherase; $P_{\text {spooA, }}$ promoter of the spo0A gene; $P_{f d x}$, promoter of the $C$. pasteurianum ferredoxin gene; SpoOA, encodes the master regulator of sporulation, SpOOA; lacZ; al pha-peptide of $\beta$-galactosidase gene containing multiple cloning sites; TT, transcriptional terminator found between hydA (hydrogenase) and pyrE. Arrows above and below the sequence labelled 1 and 2 show position of primers specific to each experiment. Lanes 1-9 (a), 1-2 (b) and 1-8 (c, d) are the screened DNA samples from randomly selected uracil prototrophic clones. a PCR screening of nine FOAR colonies using flanking primers Cac-spo0A-sF2 (1) and Cac-spo0A-sR2 (2). The expected PCR product for the mutant is $1679 \mathrm{bp}$, for the parent strain CRG1545 is $2114 \mathrm{bp}$. b PCR screening of the two uracil prototroph clones using flanking primers Cac0026SF2 (1) and Cac-hydA-sR2 (2). The expected PCR product for the pyrE mutant parent strain is $1936 \mathrm{bp}$ and for the strain in which the pyrE allele has been restored to wild-type with pMTL-ME6 is 1989 bp. c PCR screening of eight uracil prototroph using flanking primers Cac0026-sF2 (1) and Cac-hydA-sR2 (2). The expected PCR product for the parent pyrE minus strain is $1936 \mathrm{bp}$ and for the strain in which the pyrE allele has been restored to wild-type with pMTL-ME6C::spo0A is 3610 bp. d PCR screening of eight uracil prototroph using flanking primers Cac0026-sF2 (1) and Cac-hydAsR2 (2). The expected PCR product for the pyrE mutant parent strain is $1936 \mathrm{bp}$ and for the strain in which the pyrE allele has been restored to wildtype with PMTL-ME6X::spo0A is $3450 \mathrm{bp}$

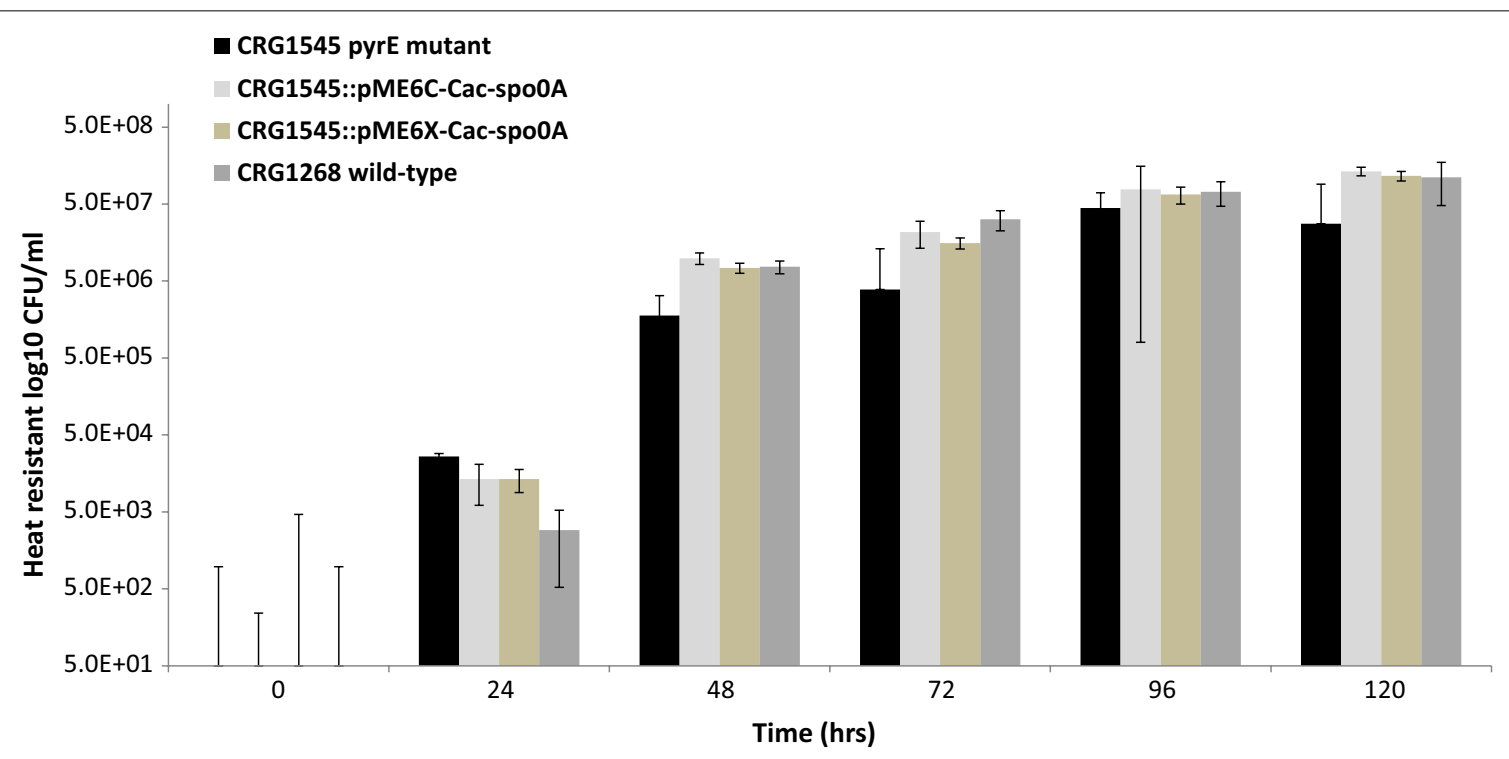

Fig. 4 Sporulation frequency of the spoOA mutant and ACE-complemented derivatives. The various strains were cultivated in CBMS medium at $37^{\circ} \mathrm{C}$ and samples taken at $0,24,48,72,96$ and $120 \mathrm{~h}$, subjected to a heat shock $\left(80^{\circ} \mathrm{C}\right.$ for $10 \mathrm{~min}$ ) before plating at serial dilutions on CBM agar and incubated for $48 \mathrm{~h}$. The number of colonies was counted and heat resistant Colony Forming Units per $\mathrm{ml}(\mathrm{CFU} / \mathrm{ml}$ ) was calculated. Estimates were undertaken in triplicate. The detection limit of the assay is $50 \mathrm{CFU} / \mathrm{ml}$, so no counts were recorded with the spo0A mutant control (not shown) or at $0 \mathrm{~h}$ with any of the strains

activity in the assay used or, more likely, more than one enzyme has such an activity.

As an alternative, the pSOL1 amyP gene (CA_P0168) was selected which is responsible for the production of an extracellular $\alpha$-amylase when grown on medium containing glucose as the sole carbon source. The expression of this gene could be easily assessed using CBM plates containing starch and stained with iodine. Loss of the pSOL1 megaplasmid, and by consequence amyP, is known to result in a loss of halo formation on medium containing starch [34]. This phenotype was confirmed by the initial generation of a ClosTron mutant, which showed a dramatic reduction in visible halo on starch iodine plates (data not shown). Accordingly, the C. acetobutylicum 824 pyrE mutant was transformed with vector pMTL-ME3:: $a m y P$ (Methods), and the in-frame deletion procedure followed exactly as described for spo0A. Three $\mathrm{FOA}^{\mathrm{R}}$ colonies were screened for the putative mutation which exhibited a reduction in halo size on starch iodine plates of an equivalent size to that seen with the ClosTron mutant. PCR screening of these clones using primers flanking the amylase gene (Cac-amyP-sF2 and Cac-amyPsR2) resulted in the expected sized (2078 bp) DNA fragment (Additional file 4: Fig. S3A). Nucleotide sequencing 
of this 2078-bp fragment confirmed the presence of the intended in-frame deletion.

A single mutant was selected and its pyrE allele was repaired, complemented and overexpressed using the ACE vectors pMTL-ME6, pMTL-ME6C::amyP and pMTL-ME6X::amyP, respectively, as described for the spoOA mutant. All the strains generated, together with the mutant and wild-type control, were grown overnight in 2x YTG broth. ODs were normalised and $20 \mu \mathrm{l}$ of each culture was spotted on CBM agar plates containing $2 \%$ starch and $0.5 \%$ glucose and incubated for $48 \mathrm{~h}$ in an anaerobic cabinet. After $48 \mathrm{~h}$, the plate was stained with iodine potassium iodide solution (Sigma 32922) for $1 \mathrm{~min}$ and photographed. The results (Fig. 5I) showed that there is no halo formation in the case of the mutant and size of halos is similar in the case of wild-type and complemented strain while slightly bigger halo was evident in the case of the overexpression strain.

\section{In-frame deletion of the $g l g A$ gene} and its complementation using pyrE

As a final test of the utility of the pyrE KO system, a mutation predicted to cause a defect in granulose accumulation was targeted. Granulose is a starch-like storage compound that is accumulated in late exponential/stationary phase prior to sporulation and acts as a carbon and energy source for subsequent endospore formation.
It is formed via the ADP-glucose pathway which requires ADP-glucose pyrophosphorylase (EC 2.7.7.27) and granulose synthase (EC 2.4.1.21) [35, 36]. The C. acetobutylicum ATCC 824 genome contains a cluster of genes involved in the glycogen formation (CA_C 2237-CA_C 2239), that includes CA_C2239 ( $g \lg A)$ predicted to encode a glycogen synthase responsible for the formation of alpha-1,4-glucan chains from ADP-glucose [29]. Accordingly, a $\mathrm{KO}$ cassette directed against $g \lg A$ was assembled and cloned into pMTL-ME3, and the resultant plasmid pMTL-ME3:: $g l g A$ used to isolate an in-frame deletion mutant in CRG1545 using the standard protocol (Methods). In this instance, PCR screening of six random $\mathrm{FOA}^{\mathrm{R}}$ clones using the flanking primers Cac-glg-sF2 and Cac-glg-sR1 generated a DNA fragment from two of a size equivalent to that obtained with the wild-type (3112 bp), while two clones generated a DNA fragment of a size (1684 bp) consistent with that which would be obtained with a deletion mutant (Additional file 4: Fig. S4). PCR failed for the remaining two clones. Nucleotide sequencing of the $1684 \mathrm{bp}$ fragment confirmed the presence of the intended in-frame deletion.

A single mutant was selected and its $p y r E$ allele repaired using pMTL-ME6, pMTL-ME6C::glgA and pMTL-ME6X::glgA, the latter two plasmids corresponding to pMTL-ME6C carrying the $g l g A$ gene together with its native promoter and pMTL-ME6X carrying
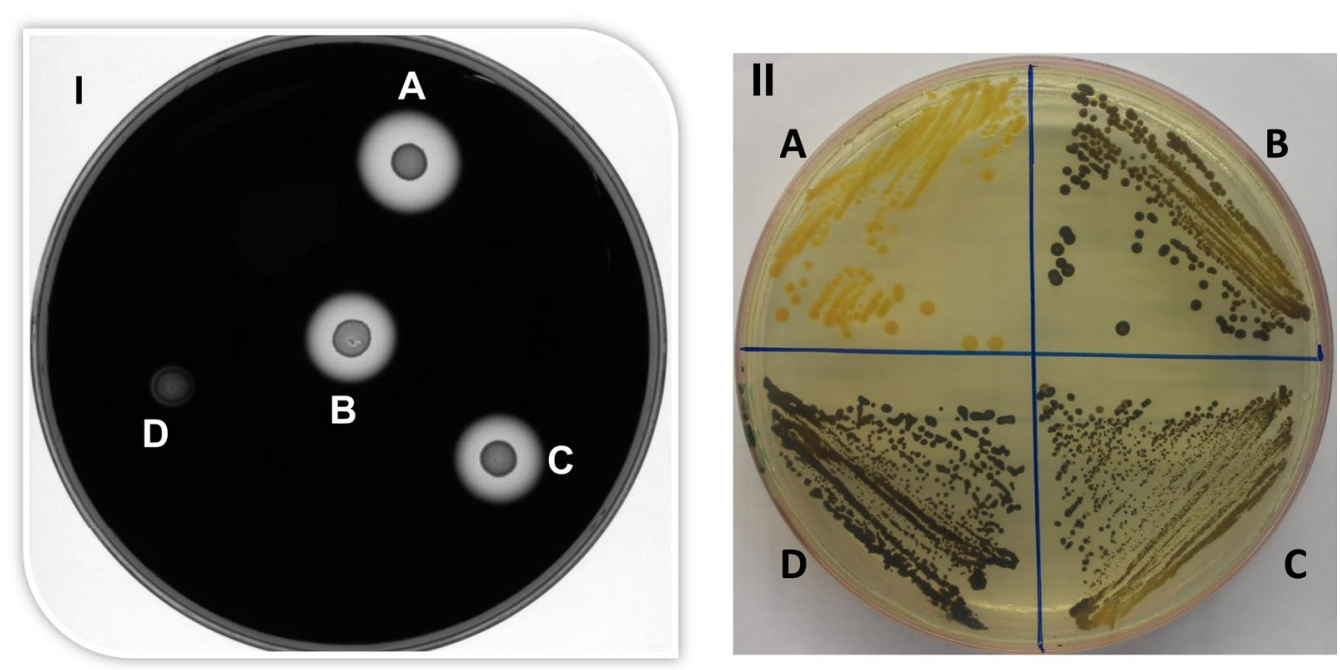

Fig. 5 Phenotypes of the amylase and granulose mutants and complemented clones. I A $20 \mu$ l aliquot of a normalised overnight culture was spotted on CBM agar containing $2 \%(\mathrm{w} / \mathrm{v})$ starch, $0.5 \%$ Glucose and plate stained with iodine potassium iodide solution after $48 \mathrm{~h}$. A CRG4897, carrying a functional copy of amy $P$ under the control of the $P_{\mathrm{fdx}}$ promoter inserted into the genome using the ACE vector pMTL-ME6X::amyP. BC. acetobutylicum ATCC 824 COSMIC strain wild-type. C CRG4896, carrying a functional copy of amyP and its own promoter inserted into the genome using the ACE vector PMTL-ME6C::amyP. D CRG4894, $\triangle$ amyP mutant. II All strains were grown on CBM agar plates with $5 \%$ glucose to promote granulose formation and exposed to iodine vapours after $72 \mathrm{~h}$. A CRG4898, $\triangle \mathrm{glg} A$ mutant; $B$ CRG4901 carrying a functional copy of glgA and its own promoter inserted into the genome using the ACE vector PMTL-ME6C::glgA; C CRG4902 carrying a functional copy of glgA under the control of the $P_{\mathrm{fdx}}$ promoter inserted into the genome using the ACE vector pMTL-ME6X::glgA. D C. acetobutylicum ATCC 824 COSMIC strain wild-type 
$g l g A$ under the control of the $\mathrm{P}_{f d x}$ promoter, respectively. The three clones generated were then tested for granulose accumulation using CBM agar plates containing $5 \%$ glucose and exposure to for $1 \mathrm{~min}$ to iodine vapour following growth for $72 \mathrm{~h}$ (Fig. 5II). These data confirmed that the $g l g A$ mutant no longer produced granulose and that its production was confirmed in both complemented strains. Any effect of placing the gene downstream of the strong $\mathrm{P}_{f d x}$ promoter was not discernible using this test.

\section{High-throughput sequencing of the pyrE and parent strains}

Having generated the pyrE mutant CRG1545 to be used as the host for future mutational studies with pMTLME3, it was important to establish that no major changes had occurred within the genome. The genome of the pyrE mutant strain (CRG1545) together with its parent strain (CRG1268) were, therefore, subjected to Illumina, singleread sequencing and the data obtained mapped to the reference GenBank sequence NC_003030 (chromosome) and NC_001988 (pSOL1) using CLC Genomics Workbench. Surprisingly, a total of 177 SNVs and 49 Indels were shared by the parent and its pyrE mutant (Additional file 2: Table S2). Aside from the presence of the expected $p y r E$ deletion, the pyrE deletion strain appeared to contain one additional SNV not present in the parent strain (Table 2). This equated to a $\mathrm{G}>\mathrm{A}$ substitution in CA_C3553 at position 3749347, which caused an Arg > Lys change at amino acid position 83 of an encoded Lacl family transcriptional regulator. The presence of this change in the pyrE mutant (CRG1545), and not the progenitor CRG1268, was confirmed by the amplification of a region of DNA encompassing the SNV and subjecting it to Sanger sequencing.

For comparative purposes, ATCC 824 was re-acquired from the ATCC (November 2011) and designated as CRG3286, genome DNA prepared and subjected to Illumina single-read re-sequencing. Of the $177 \mathrm{SNVs}$ and 49 Indels common to the ATCC 824 COSMIC consortium strain (CRG1268) and its pyrE mutant (CRG1545), 175 and 48 (Additional file 2: Table S2), respectively, were also present in the newly acquired ATCC 824 genome (CRG3286). Of the two SNVs unique to CRG1268 and CRG1545, one occurred within CA_C1534 encoding an Iron $\mathrm{ABC}$ transporter ATP-binding protein and resulted in an amino acid substitution at position 303. The other resided just upstream of CA_C3087 (phosphoenolpyruvate-protein kinase). The remaining deletion comprised a 4916-nt region of the pSOL1 megaplasmid (position 69715 to 74631, NC_001988) (Table 3). This resulted in the deletion of four genes, namely man Y/levF (mannose/ fructose-specific PTS comp. IIC), ptnd (mannose-specific PTS comp. IID), CA_P0069 (hypothetical protein) and CA_P0070 (HAD phosphatase superfamily protein) and partial deletion of ptna (mannose-specific PTS component IIAB) and CA_P0071 (xylan degrading enzyme). The region deleted is flanked by a 10-bp sequence, TGACAACCAG at positions 69705-69714 and 7462274631 , a repeat sequence that most likely mediated the 4916-bp deletion. A closer examination of the mapping data for the ATCC 824 (COSMIC) Illumina reads against the pSOL1 sequence (NC_001988) revealed that the deleted region was present, but at a very low coverage (an average read occurrence of approximately 20 compared to 400 for the remainder of pSOL1).

As the presence of the large pSOL1 deletion in the pyrE strain was not ideal, we opted to remake the $p y r E$ mutant in the re-acquired ATCC 824 strain. Accordingly, the ACE vector pMTL-JH12 was used to remake the mutant as originally described [24]. The new mutant strain was designated CRG3899 and a paired-end library constructed from its genome and re-sequenced using Illumina technology. The new strain carried the same 175 SNVs and 48 Indels common to the ATCC 824 reacquired parent strain, the COSMIC consortium strain and its pyrE mutant, but did not contain the ca. $5 \mathrm{~kb}$ deletion present in pSOL1 or the CA_C3553 SNV found in the previous pyrE mutant (CRG1545). The re-sequencing analysis of the genome indicated that there were no additional SNVs or Indels compared to the parental wild-type strain.

\section{Discussion}

In the current study we have sought to extend two methods developed for making in-frame deletions in the important human pathogen $C$. difficile to the industrially important solvent producer, $C$. acetobutylicum. Both rely on the principle of pseudo-suicide [25], in which plasmids encoding an antibiotic resistance gene (catP, specifying resistance to $\mathrm{Tm}$ ) are employed that are replication defective and limit the growth rate of the population that harbours them in the presence of antibiotic (Tm). In contrast, cells in which the plasmid has integrated by single crossover recombination grow faster and produce larger colonies, because all of the progeny carry a copy of catP. Following the isolation of the pure single cross-over integrants, cells are streaked onto media containing the counter selection agent (FOA or FC) to enable isolation of the double crossover mutant. The codA-based pMTLSC7515 uses FC, and the pyrE-based pMTL-ME3 uses FOA. As the two procedures are essentially the same, they would be expected to be equally effective in their ability to isolate mutants. Nonetheless, it generally proved easier to isolated 'pure' single-crossover, integrant colonies using pMTL-SC7515 compared to PMTL-ME3, because the larger, faster growing colonies were more readily apparent. Although both vectors employed are based on the 
unstable replicon of plasmid pIM13 [31], the growth rate of $C$. acetobutylicum carrying pMTLSC7515 in the presence of antibiotic is slightly lower than that of C. acetobutylicum carrying pMTL-ME3, and the culture achieves a lower final OD (Fig. 6). This is most likely a consequence of the larger size of pMTLSC7515 (5253 bp) compared to pMTL-ME3 (3561 bp), leading to a reduced replication efficiency.

For the isolation of double crossover mutants, the pure single crossover clones were plated onto media containing either FOA (pMTL-ME3) or FC (pMTL-SC7515). As the two plasmids are based on the same replicon and incorporate the same $\mathrm{KO}$ cassette for each targeted gene, they should be equally effective and the same number of mutants obtained. However, less screening was required to isolate mutants using PMTL-ME3 and FOA as fewer total colonies were generated suggesting that the rate of spontaneous mutants resistant to FOA was less than those that arose that were resistant to FC. Indeed, the majority of the FC colonies that arose still retained Tm resistance, even after several subcultures onto agar medium, suggesting that they retained an integrated plasmid and that spontaneous mutations elsewhere (i.e., in $\operatorname{cod} A$, upp) had led to acquisition of resistance to FC.

The primary aim of this study was the exemplification of the methods in an industrially relevant clostridia, so a number of the genes chosen for deletion had already been inactivated in the past. Thus, both systems readily allowed the isolation of in-frame deletions of spoOA (master regulator of sporulation), previously mutated using ClosTron technology and shown to abolish sporulation [13]. Similarly, an in-frame deletion of the gene

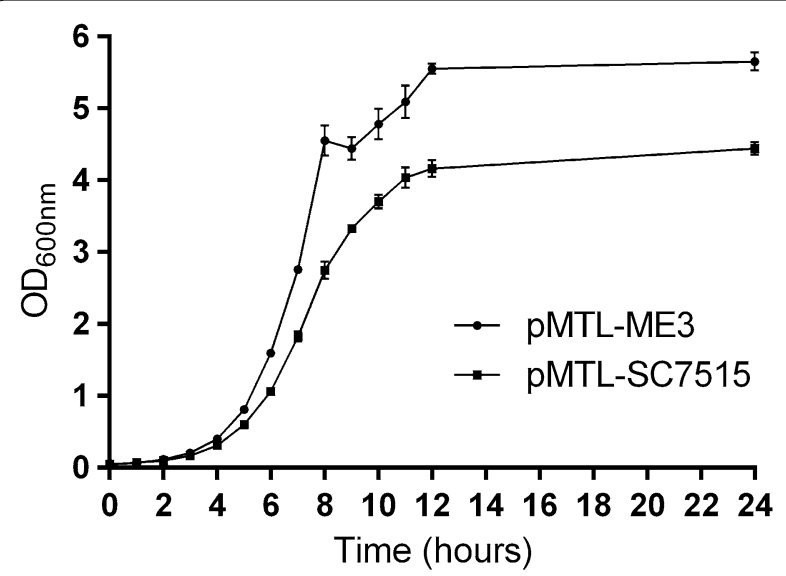

Fig. 6 Growth curve comparison of PMTL-ME3 and pMTL-SC7515. Growth of C. acetobutylicum ATCC 824 in CBMS medium supplemented with $7.5 \mu \mathrm{g} / \mathrm{ml}$ of Tm transformed with vectors pMTL-ME3 and PMTL-SC7515 with error bars representing the standard error of the mean (SEM) $(n=3)$
CA_C1502 encoding the type II restriction endonuclease cac824I was created using PMTL-SC7515 and shown, as previously [32], to allow C. acetobutylicum to be transformed with unmethylated plasmid DNA. The pyrEbased system was additionally used to inactivate for the first time a putative glycogen synthase (CA_C2239, glgA) and the pSOL1 amylase gene (CA_P0168, amyP). In both cases the expected phenotype was obtained, in terms of lack of production of granulose and amylase, respectively. As these genes had not been previously inactivated, they provided the opportunity to make use of one of the key advantages of the pyrE system, the ability to rapidly complement mutations at an appropriate gene dosage in the genome. In both cases, their $g l g A$ and amyP phenotypes were restored in terms of production of granulose and amylase.

In parallel, the opportunity was undertaken to assess the effect of overexpressing each complementing gene using the strong $\mathrm{P}_{f d x}$ promoter. There was a discernible increase in the halo around the colony in which the genome integrated copy of $a m y P$ was under the transcriptional control of $\mathrm{P}_{f d x}$ (Fig. 5I, A), in comparison to either the wild-type (Fig. 5I, B) or amyP combined with its native promoter (Fig. 5II, C). In contrast, no discernible difference could be seen in the simple plate assay used when the $g \lg A$ gene was overexpressed. This is perhaps not surprising, as unlike amylase degradation of starch, there is more than one step in granulose formation, and granulose synthase may not represent the rate limiting step. During complementation of spoOA, the effect of overexpressing spoOA using $\mathrm{P}_{f d x}$ was also explored. No increase/decrease in the number of spores made compared to when expression was from the native promoter was observed, although intriguingly, the colonies of the former were visibly larger (Fig. 5II). The reason for this change in colony morphology remains unknown.

The utility of pyrE clostridial mutants has been further demonstrated in the present study. Crucially, the mutants made are always characterised in an otherwise wild-type background as the pyrE mutant is always converted back to prototrophy, using ACE, prior to phenotypic characterisation. This is in contrast, for instance, to the use of a pyrF mutant and corresponding pyrF-based counter selection marker in C. thermocellum, where the continued presence of the mutant $p y r F$ allele causes a growth defect in the absence of exogenous uracil [19]. The C. acetobutylicum pyrE deletion strain is unable to grow at all in CMBS media, but grows at a rate that is indistinguishable from the wild-type when the media is supplemented with exogenous uracil. The mutant produces essentially the same levels of solvents as the wild-type with possibly a slight reduction in butanol levels (Additional file 4: Fig. S5). However, any reduction is of no consequence, 
as the pyrE mutation is always repaired to prototrophy, using the ACE correction vector pMTL-ME6, with restoration of wild-type solvent yields (Additional file 4: Fig. S5). More importantly, mutants created in pyrE strains (by whatever means) can be rapidly complemented using ACE vectors by the introduction of a functional copy of the inactivated gene concomitant with restoration of the pyrE allele to wild-type. The extra effort involved in the deployment of ACE vectors compared to the use of automomous complementation vectors is minimal. They require the same amount of effort in terms of construction and transfer into the desired bacterial host. Transformants carrying autonomous complementation vectors are purified by restreaking, whereas a transformant carrying an ACE complementation vector first needs to be restreaked onto a minimal plate lacking uracil, before purification by re-streaking of the selected prototrophic cell lines. The extra effort, therefore, equates to the time it takes for a uracil prototrophic colonies to develop, ca. 2 days in the case of $C$. acetobutylicum. The efficiency of ACE is such that success is assured and, moreover, cannot suffer from false positives as the nature of the pyrE deletion is such that reversion of the mutation is impossible. Although the effort required for ACE-mediated complementation is minimal, the benefits are considerable. It avoids the phenotypic effects frequently observed with high copy number plasmids and dispenses with the need to add antibiotic to ensure the retention of the complementing plasmid. Such antibiotic addition can affect phenotype and necessitate the inclusion in any phenotypic assessments of the mutant a vector only control.

The benefits of the presence of the pyrE locus are such, that there is a rational argument for using pyrE mutant hosts, and their cognate ACE correction vectors (e.g., pMTL-ME6, pMTL-ME6C and pMTL-ME6X), with any particular mutagen, including the ClosTron and any of the available negative selection markers [18-23]. Moreover, the pyrE allele represents an ideal position where other application-specific modules may be inserted, such as a sigma factor to allow deployment of a mariner transposon [37], hydrolases [38] and therapeutic genes in cancer delivery vehicles [39]. In view of these uses, it is important that one establishes that no detrimental SNVs or Indels have arisen in the genome during the construction of the pyrE host. In the case of the previously described $C$. difficile pyrE hosts, no such alterations were noted [25]. In this study a single $G>$ A substitution (Arg > Lys) was evident in the pyrE mutant in a gene (CA_C3553) annotated as a LacI family transcriptional regulator. Whilst significance of this change is unknown, its existence is immaterial since the pyrE mutant was subsequently remade in the re-acquired ATCC 824 strain during which no extra SNVs or Indels arose. This strain
(CRG3899) is, therefore, the ATCC 824 host of choice for mutational work in C. acetobutylicum.

The number of SNVs and Indels in the ATCC strain obtained from the University of Rostock, originally as the strain to be used by all consortium members of the ERANET SysMO project COSMIC, was surprising. However, upon resequencing it was apparent that the bulk of the changes were also present in the 824 deposit that currently resides at the ATCC. As a consequence it seems likely that these $175 \mathrm{SNVs}$ and 48 Indels represent mistakes in the original genome sequence, the first clostridial genome to be determined and, therefore, at a time when technologies were less sophisticated [30]. Their authenticity seems reasonably certain given that CLC Bio Workbench called these differences in four independent genome sequence runs with the DNA of the current ATCC 824, the COSMIC consortium strain and the pyrE mutants of both these strains. Moreover, in every case, correction of the identified synonymous SNVs and Indels in the ATC 824 NC_003030 and NC_001988 sequences that resided in coding regions resulted in ORFs that shared $100 \%$ identity with the equivalent encoded proteins in the genome of $C$. acetobutylicum DSM 1731. Prior to this correction, some 90 of the ATC 824 encoded proteins were not $100 \%$ identical to their DSM 1731 counterparts and in a number of instances either initiated at a different point in the genome (three) or where substantially foreshortened (twelve). Following correction of the latter changes, the C-terminal sequences of the encoding proteins were extended to give full-length sequences that were identical to the DSM 1731 homologues. In a number of instances (seven) the changes resulted in the fusion of two formerly distinct genes in the ATCC genome (NC_003030) to give a single CDS identical to that present in DSM 1731 (NC_015687). The full list of changes is given in the Additional file 2: Table S2.

A number of sequences were, however, unique to the COSMIC consortium strain. These equated to $2 \mathrm{SNVs}$ and 1 deletion. One of the SNVs occurs in the coding region of CA_C1534 (C to A, position 1678694) causing an Ala to Ser substitution in an ABC-type iron (III) transport system (ATPase component) at position 303. The second SNV is a G to A substitution at position 3241095 just upstream of CA_C3087 (phosphoenolpyruvateprotein kinase). This particular substitution resides just upstream of the predicted -10 region of the promoter of CA_C3087 within the -35 region and could conceivably effect promoter activity. All of these SNVs were confirmed as present in the COSMIC strain, and not the re-sourced ATCC 824 strain, by Sanger sequencing of appropriately PCR amplified fragments. More significant was the 4916-nt deletion within the pSOL1 megaplasmid 
(position 69715 to 74631, NC_001988) that encompassed a putative mannose PTS and related genes (Table 3). The presence of this deletion in the COSMIC consortium strain, but not the freshly sourced ATCC 824 strain, was confirmed by the PCR and subsequent sequencing of the amplified fragment using appropriate primers. A low number of reads across the deleted region were, however, present in the Illumina sequencing run suggesting that the strain represented a mixed population, in which the majority of the cells harboured a copy of the pSOL1 plasmid carrying the ca. $5 \mathrm{~kb}$ deletion. As those cells carrying the deletion appeared to be in the majority, it was not unsurprising that the selected pyrE mutant was derived from this dominant subpopulation.

During the mapping of Illumina reads derived from the ATCC 824 COSMIC strain and its pyrE mutant, but not the re-acquired ATCC 824 strain, it was noted that read coverage over a ca. $53 \mathrm{~kb}$ region from position 2016385 2069765 (in NC_003030) was some 20-fold higher (over 4000 reads) than the rest of the genome (around 200 reads) (Fig. 7). This region initiates with CA_C1867, annotated as a phage-related, Xre family transcription regulator, and ends with CA_C1957 which encodes a site specific recombinase. In between are 89 CDS encoding proteins with varying degrees of homology to proteins normally associated with bacteriophages. It suggests that during growth of the organism to prepare the DNA, the prophage most likely left the lysogenic state. Indeed, through the design of appropriate primers, it was possible to show, at least in some of the population from which the DNA was prepared, that the region had become circularised (Fig. 7). A similar increased coverage was not seen in the DNA that was prepared from the re-acquired ATCC 824.

It has previously been reported [40] that the DSM 1731 genome contains 345 single nucleotide variations (SNVs) between its chromosome and that of ATCC 824. Based on the revision to the ATCC 824 genome sequence proposed here, this would be expected to reduce that number to 170 . However, mapping of the ATCC 824, paired-end Illumina reads generated as part of this study to the DSM 1731 reference sequence

\section{Bacteriophage genome \\ Prophage F1 Prophage R2 \\ CDS

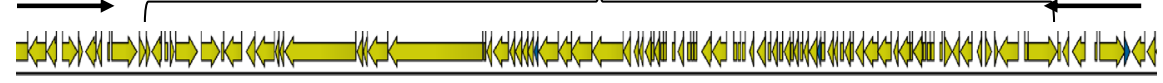

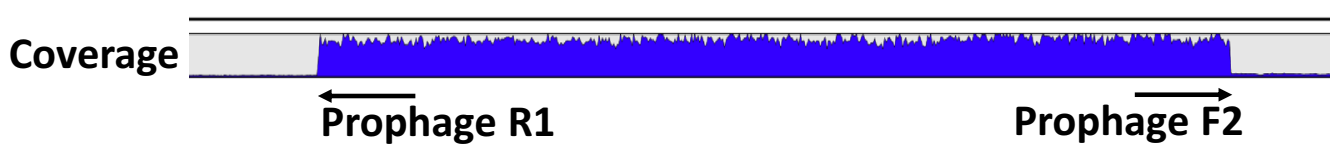

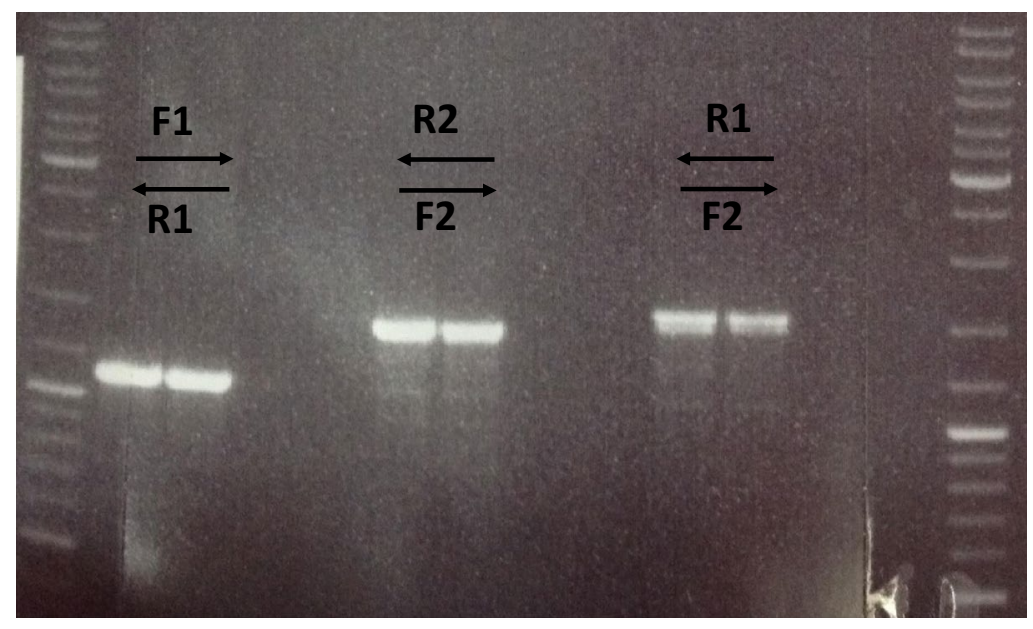

$\begin{array}{llllllllllllll}1 & 2 & 3 & 4 & 5 & 6 & 7 & 8 & 9 & 10 & 11 & 12 & 13 & 14\end{array}$

Fig. 7 Evidence for excision of a prophage from the genome of C. acetobutylicum ATCC strain COSMIC. Screen shot of the mapped paired end reads of ATCC 824 COSMIC against the NC_003030 reference sequence over the region 2016385-2069765 encompassing a prophage. The indicated primers were used to show the region was present both in the chromosome (primers prophage-F1 and prophage-R1 and prophage-F2 and prophageR2) and as an excised circular form (primers prophage-R1 and prophage-F2). The coverage for the genome was on average just over 200, whereas for the prophage encoding region it was over 4000 
NC_015687 suggests that the actual number of SNVs is 27 , with a further two deletions and two insertions, one of which is the previously reported 1753 bp insertion at position 1278525 (see Additional file 3: Table S3 for the full list). In the case of the megaplasmid of ATCC 824, pSOL1 (Table 3), our re-sequence analysis identified a total of 4 SNVs and 1 deletion that were different to the published sequence (NC_001988). All four SNVs and the single deletion were present in the megaplasmid of DSM 1731, pSMBa, suggesting that the original sequence of pSOL1 was incorrect. The deletion identified was a 4-bp deletion (ACTC) at position 191997 previously reported to distinguish $\mathrm{pSMBa}$, and the megaplasmid in C. acetobutylicum EA 2018, from pSOL1 [40]. All five changes were confirmed as correct by Sanger sequencing.

Having identified errors in both the published chromosomal (NC_003030) and pSOL1 (NC_001988) sequences, we compiled a new annotated reference sequence of both (Additional files 5, 6, respectively) that contained the corrected chromosomal and pSOL1 sequences (Additional file 5,6$)$. These sequences were then used as references for the various Illumina sequence reads generated during this project. Using CRG_003030/CRG_001988, no SNVs or Indels were called during the analysis of the reads generated from the re-acquired ATCC 824 genome, and only those expected were called when sequence reads from the two pyrE mutants and the ATCC 824 COSMIC strain were used. Coincidentally, a new SNV was identified in the COSMIC strain in CA_C1989 which was not originally identified as it agreed with the incorrect NC_003030 sequence at position 2102286. Thus, the substitution of a 'A' for 'C' at CA_C1989 position 111 resulted in replacement of a Gln with a Pro residue and an encoded Iron $\mathrm{ABC}$ transporter ATP-binding protein that is no longer identical to SMB_G2021 of DSM 1731. In addition, two SNVs were shown to be present in pSOL1 compared to $\mathrm{pSMBa}$, resulting in amino acid substitutions in two germination proteins (Table 3). These were at position 82 of an amino acid permease, Grkb1 (Gly in pSMBa, Val in pSOL1) and at position 407 of a germination receptor, GerA (Ala in pSMB, Val in pSOL1).

\section{Conclusions}

The data presented here have demonstrated that inframe deletion, marker-less mutants can be isolated in the biobutanol organism, C. acetobutylicum, through a two-stage process of single crossover integration and the subsequent isolation of double crossover excision events using replication-defective plasmids and either the counter selection marker $\operatorname{cod} A$ or $p y r E$. Either marker appears equally effective, but there is considerable merit in using a pyrE mutant as the host because, through the use of ACE vectors, mutants created in such strains (by whatever means) can be rapidly complemented. This avoids the phenotypic effects frequently observed with high copy number plasmids and dispenses with the need to add antibiotic to ensure the retention of the complementing plasmid. Our study has also revealed a surprising number of errors in the the original genome sequence of ATCC 824, while at the same time emphasising the need to re-sequence commonly used laboratory strains.

\section{Methods}

\section{Bacterial strains and routine culture conditions}

Bacterial strains and plasmids used in this study are detailed in Table 1. Escherichia coli TOP10 (Invitrogen) was cultured aerobically $\left(37^{\circ} \mathrm{C}\right.$; shaking at $\left.200 \mathrm{rpm}\right)$ in Luria-Bertani LB medium supplemented with chloramphenicol $(25 \mathrm{~g} / \mathrm{ml})$ where appropriate for plasmid cloning and storage, and TOP10 containing pAN2 plasmid [13] was used for in vivo methylation of plasmid DNA prior to transformation of $C$. acetobutylicum ATCC 824. C. acetobutylicum ATCC 824 was routinely grown anaerobically at $37{ }^{\circ} \mathrm{C}$ under an atmosphere of $\mathrm{N} 2: \mathrm{H} 2: \mathrm{CO} 2$ (80:10:10, vol:vol:vol) in an anaerobic workstation (Don Whitley, Yorkshire, UK.) using media pre-reduced overnight under the same conditions and grown in 2xYTG pH5.2 broth [41], Clostridium Basal Medium (CBM) [42] or on Clostridial Growth Medium (CGM) agar [43] supplemented with $\mathrm{Tm}(15 \mu \mathrm{g} / \mathrm{ml})$ where appropriate. Uracil was supplemented at $20 \mu \mathrm{g} / \mathrm{ml}$ or 5 -Fluoroorotic acid (FOA) (Europa Bioproducts Ltd) at $400 \mu \mathrm{g} / \mathrm{ml}$ in the media in the case of C. acetobutylicum ATCC 824 pyrE mutant. All reagents, unless noted, were purchased from Sigma-Aldrich (Tables 2, 3).

\section{Transformation}

Chemically competent cells of Escherichia coli TOP10 were prepared using $0.1 \mathrm{M} \mathrm{MgCl}_{2}$ and $0.1 \mathrm{M} \mathrm{CaCl}_{2}$ as described in [43]. Ligation mixture or $10-20 \mu \mathrm{l}$ or plasmids 1-2 $\mu \mathrm{l}$ were mixed with $50 \mu \mathrm{l}$ of chemical competent cells and incubated on ice for 10-15 min and transformed by heat shock method at $42{ }^{\circ} \mathrm{C}$ for $1 \mathrm{~min}$ in a PCR block, incubated for $1 \mathrm{~min}$ on ice followed by addition of $1 \mathrm{ml} \mathrm{LB}$ and incubating at $37{ }^{\circ} \mathrm{C}, 200 \mathrm{rpm}$ shaking for 30-60 min. Cells were centrifuged at full speed for $1 \mathrm{~min}$ and after re-suspending pellet in $200 \mu \mathrm{l}$ of LB, $100 \mu$ of cells was plated onto LB agar supplemented with chloramphenicol $(25 \mathrm{~g} / \mathrm{ml})$ and incubated overnight at $37{ }^{\circ} \mathrm{C}$. C. acetobutylicum ATCC 824 was transformed by electroporation as described by [31]. After transformation, cells were mixed in $10 \mathrm{ml}$ of $2 \mathrm{xYTG} \mathrm{pH}$ 5.2 and incubated at $37{ }^{\circ} \mathrm{C}$ for $2-3 \mathrm{~h}$ (supplemented with $20 \mu \mathrm{g} / \mathrm{ml}$ uracil in the case of pyrE mutant) followed by centrifugation at $5000 \mathrm{rpm}$ at room temperature and re-suspending the pellet in $300 \mu \mathrm{l}$ of 2 XYTG pH 5.2 and 
Table 1 Strains and plasmids used in this study

\begin{tabular}{|c|c|c|}
\hline Strain/plasmid & Relevant features & Reference/source \\
\hline \multicolumn{3}{|l|}{ Strains } \\
\hline E. coli Top10 & $\begin{array}{l}\text { F- } m c r A \Delta(\text { mrr-hsdRMS-mcrBC) } \Phi 80 l a c Z \Delta M 15 \triangle \text { lacX74 deoR recA1 araD139 } \triangle \text { (ara-leu)7697 galU } \\
\text { galKrpsL (StrR) endA1 nupG }\end{array}$ & Invitrogen \\
\hline $\begin{array}{l}\text { C. acetobutylicum } \\
\text { ATCC } 824 \text { (COSMIC) }\end{array}$ & $\begin{array}{l}\text { CRG1268 strain used in the ERANET (http://www.sysmo.net) SysMO project COSMIC. Originally } \\
\text { from G. Bennet, RICE University, USA }\end{array}$ & $\begin{array}{l}\text { H Bahl University } \\
\text { of Rostock }\end{array}$ \\
\hline $\begin{array}{l}\text { C. acetobutylicum } \\
\text { ATCC } 824\end{array}$ & CRG3286, resourced from the ATCC in November 2011 & ATCC \\
\hline $\begin{array}{l}\text { C. acetobutylicum } \\
\text { CRG1545 }\end{array}$ & $\triangle$ pyrE mutant of CRG1268 made using the ACE vector pMTL-JH12 & {$[24]$} \\
\hline $\begin{array}{l}\text { C. acetobutylicum } \\
\text { CRG3899 }\end{array}$ & $\triangle$ pyrE mutant of CRG3286 equivalent to CRG1268 & This study \\
\hline $\begin{array}{l}\text { C. acetobutylicum } \\
\text { CRG3520 }\end{array}$ & $\triangle$ spo0A of CRG1545 ( $\triangle p y r E)$ made using pMTL-ME3::spo0A & This study \\
\hline $\begin{array}{l}\text { C. acetobutylicum } \\
\text { CRG } 4889\end{array}$ & $\begin{array}{l}\triangle \text { spoOA mutant of CRG1545 generated from CRG3520 by restoration of the pyrE allele to wild- } \\
\text { type using pMTL-ME6 }\end{array}$ & This study \\
\hline $\begin{array}{l}\text { C. acetobutylicum } \\
\text { CRG } 4890\end{array}$ & $\begin{array}{l}\text { CRG3520 ( } \triangle \text { spo0A) carrying a functional copy of spo0A and its own promoter inserted into the } \\
\text { genome using the ACE vector pMTL-ME6X::spo0A concomitant with restoration of the pyrE } \\
\text { allele }\end{array}$ & This study \\
\hline $\begin{array}{l}\text { C. acetobutylicum } \\
\text { CRG4891 }\end{array}$ & $\begin{array}{l}\text { CRG3520 ( } \triangle \text { spoOA) carrying a functional copy of spoOA under the control of the } f d x \text { promoter } \\
\text { inserted into the genome using the ACE vector PMTL-ME6X::spoOA concomitant with restora- } \\
\text { tion of pyrE }\end{array}$ & This study \\
\hline $\begin{array}{l}\text { C. acetobutylicum } \\
\text { CRG4893 }\end{array}$ & $\begin{array}{l}\text { In-frame deletion mutant of amyP (CaP0168) made using pMTL-ME3::amyP (pyrE vector with } \\
\text { amyP KO cassette) }\end{array}$ & This study \\
\hline $\begin{array}{l}\text { C. acetobutylicum } \\
\text { CRG4894 }\end{array}$ & restoration of the pyrE allele to wild-type using pMTL-ME6 & This study \\
\hline $\begin{array}{l}\text { C. acetobutylicum } \\
\text { CRG4896 }\end{array}$ & $\begin{array}{l}\text { CRG4893 ( } \triangle \text { amyP) carrying a functional copy of amyP and its own promoter inserted into the } \\
\text { genome using the ACE vector pMTL-ME6C::amyP concomitant with restoration of the pyrE } \\
\text { allele }\end{array}$ & This study \\
\hline $\begin{array}{l}\text { C. acetobutylicum } \\
\text { CRG4897 }\end{array}$ & $\begin{array}{l}\text { CRG4894 ( } \triangle \text { amy } P \text { ) carrying a functional copy of amyP under the control of the } f d x \text { promoter } \\
\text { inserted into the genome using the ACE vector pMTL-ME6X::amyP concomitant with restora- } \\
\text { tion of pyrE allele }\end{array}$ & This study \\
\hline $\begin{array}{l}\text { C. acetobutylicum } \\
\text { CRG } 4883\end{array}$ & $\begin{array}{l}\text { In-frame deletion mutant of glgA (Cac2239) made using pMTL-ME3::glgA (pyrE vector with } \\
\text { glgA KO cassette) }\end{array}$ & This study \\
\hline $\begin{array}{l}\text { C. acetobutylicum } \\
\text { CRG } 4898\end{array}$ & $\begin{array}{l}\triangle g l g A \text { mutant of CRG4883 generated from CRG1545 by restoration of the pyrE allele to wild-type } \\
\text { using pMTL-ME6 }\end{array}$ & This study \\
\hline $\begin{array}{l}\text { C. acetobutylicum } \\
\text { CRG } 4901\end{array}$ & $\begin{array}{l}\text { CRG4883 }(\triangle g l g A) \text { carrying a functional copy of } g l g A \text { and its own promoter inserted into the } \\
\text { genome using the ACE vector pMTL-ME6C::glgA concomitant with restoration of the pyrE } \\
\text { allele }\end{array}$ & This study \\
\hline $\begin{array}{l}\text { C. acetobutylicum } \\
\text { CRG4902 }\end{array}$ & $\begin{array}{l}\text { CRG4883 }(\triangle g l g A) \text { carrying a functional copy of } g l g A \text { under the control of the } f d x \text { promoter } \\
\text { inserted into the genome using the ACE vector pMTL-ME6X::glgA concomitant with } \\
\text { restoration of pyrE allele }\end{array}$ & This study \\
\hline $\begin{array}{l}\text { C. acetobutylicum } \\
\text { CRG2404 }\end{array}$ & In-frame deletion mutant of spo0A gene made using pMTL-SC7515::spo0A & This study \\
\hline $\begin{array}{l}\text { C. acetobutylicum } \\
\text { CRG2403 }\end{array}$ & $\begin{array}{l}\text { In-frame deletion mutant of the type II Restriction system Cac1502 made using PMTL- } \\
\text { SC7515::cac824I }\end{array}$ & This study \\
\hline \multicolumn{3}{|l|}{ Plasmids } \\
\hline pAN-2 & E.coli vector ( $\phi 3 \mathrm{~T}$ I methyltransferase gene, tet, p15a) & CRG685 [13] \\
\hline pMTL85141 & E.coli-Clostridium Shuttle vector (pIM13 catP ColEl) & CRG1000 [31] \\
\hline pMTL-ME2 & Clostridium KO vector (pCB102 catP ColE1 pyrE) & CRG1668 [25] \\
\hline pMTL-ME3 & Clostridium KO vector (pIM13 catP ColE1 pyrE) & CRG1671This study \\
\hline pMTLSC7315 & Clostridium KO vector ( $\mathrm{pCB} 102$ catP ColE1 traJ codA) & CRG1424 [21] \\
\hline pMTLSC7515 & Clostridium KO vector (pIM13 catP ColE1 traJ codA) & CRG1303 This study \\
\hline pMTL-ME6 & C. acetobutylicum ACE correction vector (pIM13 catP ColE1 `pyrE) & CRG1861 This study \\
\hline pMTL-ME6C & C. acetobutylicum ACE complementation vector (pIM13 catP ColE1 pyrE lacZ'MCS) & CRG2483 [25] \\
\hline pMTL-ME6X & C. acetobutylicum ACE expression vector (pIM13 catP ColE1 `pyrE $P_{f d x}$, lacZ’MCS) & CRG2482 [25] \\
\hline
\end{tabular}


Table 1 continued

\begin{tabular}{llr}
\hline Strain/plasmid & Relevant features & Reference/source \\
\hline pMTL-ME3::spo0A & pMTL-ME3 + spo0A KO cassette (pIM13 catP ColE1 pyrE) & CRG1701 This study \\
pMTL-ME3::amyP & pMTL-ME3 + amyP KO cassette (pIM13 catP ColE1 pyrE) & CRG3727 This study \\
pMTL-ME3::glgA & pMTL-ME3 + glgA KO cassette (pIM13 catP ColE1 pyrE) & CRG4881 This study \\
pMTL-SC7515::Spo0A & pMTLSC7515 + spo0A KO cassette (pIM13 catP ColE1 codA) & CRG1672 This study \\
pMTL-SC7515:::Cac824l & pMTLSC7515 + cac824l KO cassette (pIM13 catP ColE1 codA) & CRG2293 This study \\
pMTL-ME6C::spo0A & ACE complementation vector for spo0A & CRG3739 This study \\
pMTL-ME6X::spo0A & ACE overexpression vector for spo0A & CRG3738 This study \\
pMTL-ME6C::amyP & ACE complementation vector for amyP & CRG3723 This study \\
pMTL-ME6X::amyP & ACE overexpression vector for amyP & CRG3722 This study \\
pMTL-ME6C::glgA & ACE complementation vector for glgA & CRG4900 This study \\
pMTL-ME6X::glgA & ACE overexpression vector for glgA & CRG4880 This study \\
\hline
\end{tabular}

Table 2 Unique SNVs and Indels, compared to the current ATCC 824 strain, in the laboratory ATCC 824 strain COSMIC and their pyrE mutants

\begin{tabular}{|c|c|c|c|c|c|c|c|c|c|}
\hline Strain & NC_003030 & Type & Ref & Allele & Feature & Change & AA Change & Function & CRG_003030 \\
\hline \multirow[t]{3}{*}{ ATCC 824 COSMIC } & 1678711 & SNV & C & A & CA_C1534 & $907 \mathrm{G}>\mathrm{T}$ & Ala303Ser & $\begin{array}{l}\text { Iron } A B C \text { transporter } \\
\text { ATP-binding protein }\end{array}$ & 1678691 \\
\hline & $2102314^{a}$ & SNV & $G(T)$ & G & CA_C1989 & $332 A>C$ & Gln111Pro & Iron (III) ABC transporter ATPase & 2102286 \\
\hline & 3241117 & SNV & G & $\mathrm{T}$ & Miscellaneous & - & - & Promoter region of CA_C3087 & 3241092 \\
\hline ATCC 824 COSMIC PyrE & 3749367 & SNV & G & A & CA_C3553 & $248 G>A$ & Arg83Lys & $\begin{array}{l}\text { Lacl family transcriptional } \\
\text { regulator }\end{array}$ & 3749536 \\
\hline
\end{tabular}

a sequence identical to CA_C1989 in NC_003030 annotation at position 2102314 (G), but once the sequence is corrected to a 'T', the COSMIC 'G' at this position becomes a SNV

${ }^{\text {b }}$ CRG_003030.gbk is a corrected annotation of the ATTC 824 chromosomal genome sequence and is available as a Additional file 5

Table 3 SNVs in the pSOL1 megaplasmid of C. acetobutylicum ATCC 824 COSMIC strain and re-acquired ATCC 824 strain

\begin{tabular}{|c|c|c|c|c|c|c|c|}
\hline NC_001988 & Type & Ref & Allele & Feature & Change & AA Change & Function/comment \\
\hline \multicolumn{8}{|c|}{ ATCC 824 isolate re-acquired from the ATCC in November 2011} \\
\hline 18758 & SNV & G & T & CA_P0017 & $11 \mathrm{C}>\mathrm{A}$ & Ala4Glu & GrkB germination protein, 4Glu also in SMB_P016 of DSM 1731 \\
\hline 54266 & SNV & A & G & CA_P0053 & $317 \mathrm{~T}>\mathrm{C}$ & Leu106Pro & Xylanase XYNB, now identical to SMB_P052 of DSM 1731 \\
\hline 56219 & SNV & A & G & CA_P0055 & $506 \mathrm{~A}>\mathrm{G}$ & Asn169Ser & Hypothetical protein, now identical to SMB_P054 of DSM 1731 \\
\hline 60555 & SNV & G & $\mathrm{T}$ & CA_P0059 & $754 C>A$ & His252Asn & Alcohol dehydrogenase, now identical to SMB_P058 of DSM 1731 \\
\hline 191997 & Del & ACTC & - & Deletion & - & - & Same deletion as in pSMBa of DSM1731 \\
\hline \multicolumn{8}{|c|}{ ATCC 824 COSMIC strain } \\
\hline 18758 & SNV & G & $\mathrm{T}$ & CA_P0017 & $11 \mathrm{C}>\mathrm{A}$ & Ala4Glu & GrkB germination protein, 4Glu also in SMB_P016 of DSM 1731 \\
\hline 54266 & SNV & A & G & CA_P0053 & $317 \mathrm{~T}>\mathrm{C}$ & Leu106Pro & Xylanase XYNB, now identical to SMB_P052 of DSM 1731 \\
\hline 56219 & SNV & A & G & CA_P0055 & $506 \mathrm{~A}>\mathrm{G}$ & Asn169Ser & Hypothetical protein, now identical to SMB_P054 of DSM 1731 \\
\hline 60555 & SNV & G & $\mathrm{T}$ & CA_P0059 & $754 C>A$ & His252Asn & Alcohol dehydrogenase, now identical to SMB_P058 of DSM 1731 \\
\hline 69715 & Del & 69715. &. .74631 & CA_P0066-71 & del4916 bp & - & $\begin{array}{l}\text { Unique to pSOL1, affects ptna, manY,ptnd, CA_P0069, CA_P0070, } \\
\text { CA_p0071 }\end{array}$ \\
\hline 191997 & Del & ACTC & - & Deletion & - & - & Same deletion as in pSMBa of DSM1731 \\
\hline \multicolumn{8}{|c|}{ DSM 1731 (map positions are to NC_015686) } \\
\hline 18524 & SNV & C & A & SMB_P016 & $245 G>T$ & Gly82Val & $\begin{array}{l}\text { GrkB, germination protein, Gly at position } 82 \text { instead of Val in CA_ } \\
\text { P0017 }\end{array}$ \\
\hline 20626 & SNV & G & A & SMB_P019 & $220 \mathrm{C}>\mathrm{T}$ & Ala407Val & $\begin{array}{l}\text { GrkA, germination receptor, Ala at position } 407 \text { instead of Val in } \\
\text { CA_P0020 }\end{array}$ \\
\hline
\end{tabular}


plating $100 \mu$ l onto CGM agar supplemented with $15 \mu \mathrm{g}$ / $\mathrm{ml} \mathrm{Tm}$ and incubated at $37^{\circ} \mathrm{C}$ in anaerobic conditions for $24-48 \mathrm{~h}$.

\section{Cloning and PCR analysis}

C. acetobutylicum ATCC 824 and C. sporogenes ATCC 15579 genomic DNAs for use in cloning and PCR analysis were prepared using QIAGEN DNeasy Blood and Tissue Kit in accordance with the manufacturer's instructions and recommended pretreatment for Grampositive bacteria using Lysozyme from chicken egg white (Cat L6876 Sigma-Aldrich) $20 \mathrm{ml} / \mathrm{ml}$ phosphate buffer saline (PBS) [25]. Screening PCRs were performed using DreamTaq ${ }^{\text {TM }}$ green PCR master mix (Fisher Scientific UK Ltd) and Failsafe ${ }^{\mathrm{TM}}$ PCR system (Epicentre) for downstream cloning in accordance with the manufacturer's instructions. Primers were designed using web tool available at http://primer3.ut.ee and are listed in Additional file 1: Table S1. All the DNA manipulations including restriction digestion, de-phosphorylation of $5^{\prime}$ end and ligation were carried out according to standard procedures as described in [44]. DNA extraction and purification from agarose gel and PCR reaction mixtures were undertaken using QIAquick Gel Extraction and PCR Purification Kit (Qiagen Ltd UK), respectively, and used according to the manufacturer's instructions.

\section{Vector construction}

The $C$. difficile knock-out (KO) vectors pMTL-YN3/ pMTL-ME2 (pyrE) and pMTL-SC7315 $(\operatorname{codA})$ are based on the pCB102 plasmid replication region [21]. As plasmids that utilise the pIM13 replicon have proven highly effective as integration vectors in C. acetobutylicum, the pCB102 replication regions of pMTL-ME2 and PMTL-SC7315 were replaced with that of pIM13. Both pMTL-ME2 and pMTL-SC7315 correspond to the pMTL80000 modular format [31]. Their Gram-positive replicons are, therefore, flanked by AscI and FseI restriction sites. Accordingly, their pCB102 replicons were excised by cleavage with AscI and FseI and replaced with $878 \mathrm{bp} \mathrm{AscI/FseI} \mathrm{fragment} \mathrm{from} \mathrm{the} \mathrm{modular} \mathrm{plas-}$ mid pMTL85141 [31] that encompassed the pIM13 replication region. The two new vectors were designated pMTL-ME3 (derived from pMTL-ME2) and pMTLSC7515 (derived from pMTL-SC7315). For chromosomal complementation and overexpression at the pyrE locus in mutants made using pMTL-ME3, plasmids pMTLME6C and pMTL-ME6X were used, respectively [25]. For the ACE-mediated correction of the pyrE locus of CRG1545 back to wild-type, pMTL-ME6 a functional equivalent to pMTL-YN1 was made [25]. A fragment of $1850 \mathrm{bp}$, encompassing regions of pyrE and hydA genes, was cloned by PCR using primers pyrE-LHAv1.0-F1 and
hydA-RHAv1.0-R2 from C. acetobutylicum genomic DNA and was digested with HindIII and BglII and a 939bp fragment was purified from agarose gel and ligated into pMTL-JH14 [24] linearized with the same restriction enzymes and confirmed by restriction analysis and Sanger sequencing.

\section{KO plasmids}

Allele exchange cassettes specific to target loci were either constructed by SOE PCR [45] or commercially synthesised. In every case the authenticity of each final plasmid was verified by restriction digestion and nucleotide sequencing. Each cassette was composed of a lefthomology arm (LHA) and a right-homology arm (RHA), each $750 \mathrm{bp}$ in size. The spoOA KO cassette was designed to make an in-frame deletion that removed $435 \mathrm{bp}$ out of $846 \mathrm{bp}$ and was composed of the C. acetobutylicum bases 2171926 to 2172675 (LHA) fused to bases 2173111 to 2173860 (RHA) by SOR PCR. The flanking primers used added GTTTAAAC (a PmeI recognition sequence) at each extremity allowing the 1500-bp fragment to be blunt-end cloned into TA cloning vector pCR2.1 3929 bp and subsequently re-isolated as a BamHI/NotI fragment and inserted between the BamHI and NotI sites of pMTL-ME3 and PMTL-SC7515 to yield plasmid PMTLME3::spo0A and pMTLSC7515::spo0A, respectively. The KO cassette for CA_P0168 (amyP) was designed to delete 2277 bp of the 2283 bp long gene and comprised the C. acetobutylicum bases 180839 to 181588 (LHA) fused to bases 183866 to 184615 (RHA). It was synthesised by Eurofine www.eurofinsgenomics.eu and was flanked by BamHI and NcoI restriction sites. Following cleavage by BamHI and NcoI it was inserted between the equivalent restriction sites of pMTL-ME3 to give pMTL-ME3::amyP. The KO cassette targeting the $g l g A$ gene was composed of bases 2332450 to 2333199 (LHA) of the C. acetobutylicum ATCC 824 genome fused bases 2334628 to 2335377 (RHA) and was designed to remove $1428 \mathrm{bp}$ of the $1434 \mathrm{bp}$ long gene. The PCR primers used were designed to position a NotI restriction recognition site adjacent to the LHA and a PmeI site at the extremity of the RHA. The cassette was isolated as a 1500-bp NotI/PmeI fragment and inserted between the NotI site and NheI (blunt-ended with T4 polymerase) sites of pMTL-ME3 to yield the KO plasmid pMTL-ME3::glgA. The in-frame deletion cassette for CA_C1502 (cac824I) consisted of the C. acetobutylicum bases 1645573 to 1646322 (LHA) fused to 1647031 to 1647780 (RHA), which removes $708 \mathrm{bp}$ from the $714 \mathrm{bp}$ long gene. PmeI restriction sites were introduced as part of the SOE PCR flanking primers in the LHA and RHA which allowed the 1500-bp fragment to be isolated following digestion with PmeI and inserted into the equivalent site of 
plasmid pMTLSC7515 to resultant in the $\mathrm{KO}$ plasmid pMTL-SC7515::cac824I.

\section{Complementation vectors}

To complement those genes knocked out using the $p y r E$ system, appropriate DNA fragments encompassing the structural gene and native promoter were cloned into the ACE vector pMTL-ME6C. The spoOA gene with its native promoter was amplified as a 1211-bp fragment using primers NotI-Cacspo0A-F1 and EcoRI-Cacspo0A$\mathrm{R} 1$ and following appropriate digestion cloned between the NotI and EcoRI sites of pMTL-ME6C to result in the plasmid pMTL-ME6C::spo0A. Similarly, the C. acetobutylicum ATCC 824 amyP gene (CA_P0168) was PCR amplified using primers Pca_P-0168-F1 and Ca_P0168-R1 and the 2505-bp PCR product cleaved with NotI and BamHI and inserted between the equivalent sites of pMTL-ME6C. The plasmid obtained was designated pMTL-ME6C::amyP. To bring about overexpression of the complementing gene, promoter-less copies were cloned into pMTL-ME6X downstream of the strong $\mathrm{P}_{\mathrm{fdx}}$ promoter. The spo0A gene was PCR amplified from genomic DNA using primers NdeI-Cacspo0A-F1 and EcoRI-Cacspo0A-R1. The PCR product obtained (859 bp) was digested with NdeI and EcoRI and ligated downstream of the $\mathrm{P}_{\mathrm{fdx}}$ promoter in pMTLME6X linearized with the same restriction enzymes, to make pMTL-ME6X::spo0A.

To construct an over-expression vector for $a m y P$, the gene was PCR amplified from genomic DNA without its promoter using $\mathrm{Ca}_{-} \mathrm{P}-0168-\mathrm{F} 2$ and Ca_P-0168-R1. The start codon of the gene was changed from TTG to ATG using AseI in the forward primer which was compatible with NdeI. The 2300-bp PCR product obtained was digested with AseI and BamHI and cloned in pMTL-ME6X linearized with NdeI and BamHI to make pMTL-ME6X::amyP. However, cells transformed with this plasmid were negative for amylase activity by the starch-iodine plate test. To remedy this lack of expression, the ATG was changed back to the original TTG start by making a quick change in the vector using oligonucleotides Qfdx-0168-F1 and Qfdx-0168-R1, while at the same time reducing the spacer region between the start codon and ribosome binding site by one base pair.

\section{Allelic exchange procedure}

The procedure used for the isolation of the in-frame deletion mutants in $C$. acetobutylicum using either pMTL-ME3 or pMTL-SC7515 is essentially the same and based on the procedures described by $\mathrm{Ng}$ el et al. [25] and Cartman et al. [21], respectively. Derivatives of the two vectors carrying the required $\mathrm{KO}$ cassette were electroporated into either the wild-type (pMTL-SC7515) or the pyrE mutant strain (pMTL-ME3) and plated on CGM supplemented with $15 \mu \mathrm{g} / \mathrm{ml} \mathrm{Tm}$ and incubated for 24-48 h. The largest representative colonies were selected and passaged twice by re-streaking onto the same agar media, at each stage choosing larger representative colonies. The cells of selected colonies were then screened by PCR to ascertain whether the isolated clonal population was a pure single crossover integrant, using appropriate pairs of primers. These comprised a forward primer complementary to the chromosome and a reverse primer specific to a plasmid encoded region. The presence of a PCR product indicated that the clones were single crossover integrants, and the size of PCR product indicated at which homology arm (LHA or RHA) recombination had happened. It was important to establish purity, as the presence of cells in which integration had not occurred would contribute to a high background level of colonies when subsequently plated on the counter selection agents, FOA and FC.

Pure single, crossover clones were plated onto CGM supplemented with $20 \mu \mathrm{g} / \mathrm{ml}$ uracil or un-supplemented CGM medium and incubated for 2-3 days to allow cells to undergo a second recombination event, resuspended in PBS and appropriate dilutions spread on CGM agar plates supplemented with either FOA $(400 \mu \mathrm{g} / \mathrm{ml})$ and uracil $(1 \mu \mathrm{g} / \mathrm{ml})$ or CBM supplemented with FC $(100 \mu \mathrm{g} /$ $\mathrm{ml}$ ) for pMTL-ME3 and pMTL-SC7515, respectively, and incubated for $48 \mathrm{~h}$. Only cells that have, respectively, lost pyrE or $\operatorname{cod} A$ through excision of the plasmid and its subsequent loss from the cell may grow. The loss of the KO vector was confirmed by patch plating on CGM with or without Tm supplementation. As the excision event may be mediated by either of the two homology arms (LHA or RHA), the cell line that arises can be either mutant or wild-type. Where there is no homology arm preference, then the ratio of mutant to wild-type should be 50:50. However, this is rarely the case. Moreover, if one homology arm is favoured at the single crossover integration stage, then it will be favoured at the double crossover stage and as a consequence the proportion of wild-type derivatives will be higher.

Screening of $\mathrm{FC}^{\mathrm{R}}$ and $\mathrm{FOA}^{\mathrm{R}}$ clones by PCR was undertaken using primer pairs that flanked the desired deletion point and were complementary to chromosomally located sequence that resided external to the homology arms used. For example, Cac-spoOA-sF2 and Cacspo0A-sR2 in the case of spo0A; Cac-1501-sF2 and Cac-1504-sR1 in the case of cac824I; Cac-amyP-sF2 and Cac-amyP-sR2 in the case of $a m y P$ and; Cac-glg-sF2 and Cac-glg-sR1 in the case of $g l g A$. The PCR products generated were subjected to Sanger sequencing to confirm their authenticity. 


\section{Correction of pyrE using ACE}

The ACE procedure adopted is essentially as described before [24]. Plasmids pMTL-ME6 and the derivatives of pMTL-ME6C and pMTL-ME6X prepared from E. coli pAN2 were transformed into the appropriate $C$. acetobutylicum mutant and $\mathrm{Tm}^{\mathrm{R}}$ transformants selected onto CGM agar supplemented with $15 \mu \mathrm{g} / \mathrm{ml} \mathrm{Tm}$ and $20 \mu \mathrm{g} /$ $\mathrm{ml}$ uracil. Selected large colonies (single crossover integrants) were then streaked onto CBM lacking uracil supplementation to select plasmid excised derivatives (double crossover cells) in which the pyrE allele had been restored to wild-type and the cells had become prototrophic. PCR primers Cac0026-sF2 and Cac-hydA-sR2 were used to screen for authentic clones and the amplified fragment subject to nucleotide sequencing.

\section{High-throughput sequencing}

Phenol chloroform method was used to isolate genomic DNA from strains CRG1268 (COSMIC wild-type strain), CRG1545 (COSMIC strain pyrE mutant), CRG3286 (reacquired ATCC 824) and CRG3899 (re-acquired ATCC 824 pyrE mutant). CRG1268, CRG1545 and CRG3286 were sequenced by Illumina hiseq (GATC, Germany) with read lengths of 101, 51 and 51, respectively. For the sequencing of CRG3899 paired-end libraries were prepared and sequenced (251 bp reads) using an Illumina MiSeq benchtop sequencer (Deepseq, University of Nottingham). Preparation of paired-end libraries as well as sequencing was performed as described by the manufacturers. Sequence reads were mapped to the reference sequences NC_003030 (chromosome) and NC_001988 (pSOL1) using CLC Genomics Workbench. We used a frequency of $90 \%$ as a cut-off for SNV calling. Selected SNVs and InDels were confirmed by amplifying a few hundred base pairs up- and downstream of the area of interest and the amplicon was Sanger sequenced (Source BioScience, UK).

\section{Sporulation assay}

Spore stocks of test strains were heat treated at $80{ }^{\circ} \mathrm{C}$ for $10 \mathrm{~min}$ before plating on pre-reduced CBM agar and incubation for $24 \mathrm{~h}$. Triplicate pre-cultures were prepared by resuspending a loop-full of colonies from each strain into three universal tubes containing $10 \mathrm{ml}$ CBMS medium (CBM $+0.5 \% \mathrm{w} / \mathrm{v} \mathrm{CaCO}_{3}, 5 \% \mathrm{w} / \mathrm{v}$ glucose) and incubated overnight. The following day, triplicate sporulation assay cultures (30 ml CBMS medium in $50 \mathrm{ml}$-Falcon tubes) were inoculated to an initial $\mathrm{OD}_{600}$ $\mathrm{nm}$ 0.048-0.0065 from the pre-cultures. Aliquots of $200 \mu \mathrm{l}$ were removed at $0,24,48,72,96$, and $120 \mathrm{~h}$. Samples were heated at $80{ }^{\circ} \mathrm{C}$ for $10 \mathrm{~min}$ in a water bath and dilutions $\left(10^{-1}-10^{-6}\right)$ were prepared in 96 well microtitre plate. From each strain, $20 \mu \mathrm{l}$ volumes were spotted onto CBM agar and incubated for $48 \mathrm{~h}$. The number of colonies was counted and heat_resistant Colony Forming Units per $\mathrm{ml}(\mathrm{CFU} / \mathrm{ml})$ was calculated.

\section{Detection of granulose}

Granulose accumulation was tested by growing the $C$. acetobutylicum ATCC 824 strains on agar-solidified CBM containing $5 \%$ glucose to promote granulose accumulation. After incubation for $72 \mathrm{~h}$, plates were exposed to iodine vapour, staining granulose-containing colonies dark brown.

\section{Additional files}

Additional file 1: Table S1. This file contains all oligonucleotides used in this study.

Additional file 2: Table S2. This file contains full details of SNV and Indels of ATCC 824 against the GenBank ATCC 824 sequence, NC_003030.

Additional file 3: Table S3. This file contains full details of the SNVs and Indels of ATCC 824 Illumina reads against the DSM 1731 GenBank genome sequence, NC_015686.

Additional file 4. Supplementary Figures S1, S2, S3 and S4.

Additional file 5. Corrected annotated chromosomal genome sequence of ATCC 824.

Additional file 6. Corrected annotated sequence of the $\mathrm{PSOL} 1$ megaplasmid of ATCC 824.

\section{Abbreviations}

ACE: allele-Coupled Exchange; CBM: clostridial basal medium; CGM: clostridial growth medium; FC: 5-fluorocytosine; FOA: 5-fluorouracil; Indels: insertions and deletions; KO: knock-out; SNV: single nucleotide variations; SOE: splicing by overlap extension; Tm: thiamphenicol.

\section{Authors' contributions}

ME carried out the majority of the plasmid molecular biology, mutant creation and phenotypic analysis and helped draft the manuscript. STC and YZ made plasmid PMTL-SC7515 and the mutant strain CRG3899, respectively, and helped to revise the manuscript. WK, ME and YZ undertook the genome resequencing and analysis and helped to revise the manuscript. JTH designed and oversaw plasmid construction and experiments in the initial part of the study and helped to revise the manuscript. NPM conceived the study, oversaw its design and coordination, helped with the genome sequence analysis and drafted the manuscript. KW participated in the design and coordination of the study and helped to revise the manuscript. All authors read and approved the final manuscript.

\section{Author details}

${ }^{1}$ Clostridia Research Group, BBSRC/EPSRC Synthetic Biology Research Centre (SBRC), University of Nottingham, University Park, Nottingham NG7 2RD, UK. 2 Present Address: MicCell Bioservices B.V., Edisonstraat 101, 7006 RB Doetinchem, The Netherlands. ${ }^{3}$ Present Address: Department of Life Sciences, Centre for Synthetic Biology and Innovation, Imperial College London, South Kensington Campus, London SW7 2AZ, UK. ${ }^{4}$ Present Address: Intermediates Sustainability, INVISTA Intermediates, Wilton Centre, Redcar TS10 4RF, UK.

\section{Acknowledgements}

This work was undertaken as part of the activities of the BBSRC Sustainable Bioenergy Centre (BESBEC) under grant agreement number BB/G016224/1 and the BBSRC/EPSRC Synthetic Biology Research Centre, Grant Agreement No BB/L013940/1. ME acknowledges the support of TMO Renewables Ltd.

\section{Competing interests}

The authors declare that they have no competing interests. 
Received: 19 August 2015 Accepted: 4 December 2015

Published online: 04 January 2016

\section{References}

1. Durre P. Handbook on Clostridia. CRC Press, 2005.

2. Stevens DL, Aldape MJ, Bryant AE. Life-threatening clostridial infections. Anaerobe. 2012;18:254-9.

3. Cho C, Jang Y-S, Moon HG, Lee J, Lee SY. Metabolic engineering of clostridia for the production of chemicals. Biofuels Bioprod Biorefin. 2015;9:211-25.

4. Keis S, Bennett CF, Ward VK, Jones DT. Taxonomy and phylogeny of industrial solvent-producing clostridia. Int J Syst Bacteriol. 1995;45(4):693-705

5. Jones DT, Woods DR. Acetone-butanol fermentation revisited. Microbiol Rev. 1986;50(4):484-524.

6. Lütke-Eversloh T. Application of new metabolic engineering tools for Clostridium acetobutylicum. Appl Microbiol Biotechnol. 2014;98(13):5823-37.

7. Green EM, Bennett GN. Inactivation of an aldehyde/alcohol dehydrogenase gene from Clostridium acetobutylicum ATCC 824. Appl Biochem Biotechnol. 1996;57(58):213-21.

8. Green EM, Boynton ZL, Harris LM, Rudolph FB, Papoutsakis ET, Bennett GN. Genetic manipulation of acid formation pathways by gene inactivation in Clostridium acetobutylicum ATCC 824. Microbiol. 1996;142(8):2079-86.

9. Nair RV, Green EM, Watson DE, Bennett GN, Papoutsakis ET. Regulation of the sol locus genes for butanol and acetone formation in Clostridium acetobutylicum ATCC 824 by a putative transcriptional repressor. J Bacteriol. 1999;181(1):319-30.

10. Wilkinson SR, Young M. Targeted gene integration of genes into the Clostridium acetobutylicum chromosome. Microbiol. 1994;140:89-95.

11. Chen Y, Caruso L, McClane B, Fisher D, Gupta P. Disruption of a toxin gene by introduction of a foreign gene into the chromosome of Clostridium perfringens using targetron-induced mutagenesis. Plasmid. 2007;58:182-9.

12. Shao L, Hu S, Yang Y, Gu Y, Chen J, Yang Y, Jiang W, Yang S. Targeted gene disruption by use of a group II intron (targetron) vector in Clostridium acetobutylicum. Cell Res. 2007;17:963-5.

13. Heap JT, Pennington OJ, Cartman ST, Carter GP, Minton NP. The ClosTron: a universal gene knock-out system for the genus Clostridium. J Microbiol Methods. 2007;70:452-64

14. Heap JT, Kuehne SA, Ehsaan M, Cartman ST, Cooksley CM, Scott JC, Minton NP. The ClosTron: mutagenesis in Clostridium refined and streamlined. J Microbiol Methods. 2010;80:49-55.

15. Kuehne SA, Heap JT, Cooksley CM, Cartman ST, Minton NP. ClosTronmediated engineering of Clostridium. Meth Mol Biol. 2011;765:389-407.

16. Kuehne SA, Minton NP. ClosTron-mediated engineering of Clostridium. Bioengineered. 2012;3(4):247-54.

17. Liu Y-J, Zhang J, Cui G-Z, Cui Q. Current progress of targetron technology: development, improvement and application in metabolic engineering. Biotechnol J. 2015;10:855-65.

18. Nariya H, Miyata S, Suzuki M, Tamai E, Okabe A. Development and application of a method for counterselectable in-frame deletion in Clostridium perfringens. Appl Environ Microbiol. 2011;77:1375-82.

19. Tripathi SA, Olson DG, Argyros DA, Miller BB, Barrett TF, Murphy DM, McCool JD, Warner AK, Rajgarhia VB, Lynd LR, Hogsett DA, Caiazza NC. Development of pyrF-based genetic system for targeted gene deletion in Clostridium thermocellum and creation of a pta mutant. Appl Environ Microbiol. 2010;76:6591-9.

20. Dusséaux S, Croux C, Soucaille P, Meynial-Salles I. Metabolic engineering of Clostridium acetobutylicum ATCC 824 for the high-yield production of a biofuel composed of an isopropanol/butanol/ethanol mixture. Metab Eng. 2013;18:1-8.

21. Cartman ST, Kelly ML, Heeg D, Heap JT, Minton NP. Precise manipulation of the Clostridium difficile chromosome reveals a lack of association between the $t c d C$ genotype and toxin production. Appl Environ Microbiol. 2012;78(13):4683-90.

22. Al-Hinai MA, Fast AG, Papoutsakis ET. Novel system for efficient isolation of Clostridium double-crossover allelic exchange mutants enabling markerless chromosomal gene deletions and DNA integration. App Environ Microbiol. 2014;78:8112-21.

23. Argyros DA, Tripathi SA, Barrett TF, Rogers SR, Feinberg LF, Olson DG, Foden JM, Miller BB, Lynd LR, Hogsett DA, Caiazza NC. High ethanol titers from cellulose by using metabolically engineered thermophilic, anaerobic microbes. Appl. Environ. Microbiol. 2011;77:8288-94.

24. Heap JT, Ehsaan M, Cooksley CM, Ng YK, Cartman ST, Winzer K, Minton NP. Integration of DNA into bacterial chromosomes from plasmids without a counter-selection marker. Nucl Acids Res. 2012;0(8):e59.

25. Ng YK, Ehsaan M, Philip S, Collery MM, Janoir C, Collignon A, Cartman ST, Minton NP. Expanding the repertoire of gene tools for precise manipulation of the Clostridium difficile genome: allelic exchange using pyrE alleles. PLoS One. 2013:8:e56051.

26. Minton N, Morris JG. Isolation and Partial Characterization of 3 Cryptic Plasmids from Strains of Clostridium-Butyricum. J Gen Microbiol. 1981;127:325-31.

27. Abraham LJ, Wales AJ, Rood JI. Worldwide distribution of the conjugative Clostridium perfringens tetracycline resistance plasmid, pCW3. Plasmid. 1985;14:37-46.

28. Heidelberger C, Danenberg PV, Moran RG. Fluorinated pyrimidines and their nucleosides. Adv Enzymol Relat Areas Mol Biol. 1983;1983(54):58-119.

29. Longley DB, Harkin DP, Johnston PG. 5-Fluorouracil: mechanisms of action and clinical strategies. Nat Rev Cancer. 2003;2003(3):330-8.

30. Nolling J, Breton G, Omelchenko MV, Makarova KS, Zeng QD, Gibson R, Lee HM, Dubois J, Qiu DY, Hitti J, Wolf YI, Tatusov RL, Sabathe F, Doucette-Stamm L, Soucaille P, Daly MJ, Bennett GN, Koonin EV, Smith DR, Finishing GSCP. Genome sequence and comparative analysis of the solvent-producing bacterium Clostridium acetobutylicum. J Bacteriol. 2001;183(16):4823-8

31. Heap JT, Pennington OJ, Cartman ST, Minton NP. A modular system for Clostridium shuttle plasmids. J Microbiol Methods. 2009;8:79-85.

32. Dong H, Zhang Y, Dai Z, Li Y. Engineering Clostridium strain to accept unmethylated DNA. PLoS One. 2010;5(2):e9038.

33. Soucaille P, Figge R, Croux C. Process for chromosomal integration and DNA sequence replacement in Clostridia. 2006. International Patent W02008/0.

34. Sabathe F, Croux C, Cornillot E, Soucaille P. amyP, a reporter gene to study strain degeneration in Clostridium acetobutylicum ATCC 824. FEMS Microbiol Lett. 2002;210(1):93-8.

35. Alsaker KV, Papoutsakis ET. Transcriptional program of early sporulation and stationary-phase events in Clostridium acetobutylicum. J Bacteriol. 2005; 187:7103-18.

36. Steiner EJ, Scott JC, Minton NP, Winzer K. An agr quorum sensing system that regulates granulose formation and sporulation in Clostridium acetobutylicum. Appl Environ Microbiol. 2012;78(4):1113-22.

37. Zhang Y, Grosse-Honebrink A, Minton NP. A universal mariner transposon system for forward genetic studies in the genus Clostridium. PLoS One. 2015;2;10(4):e0122411.

38. Kovács K, Willson BJ, Schwarz K, Heap JT, Jackson A, Bolam DN, Winzer K, Minton NP. Secretion and assembly of functional mini-cellulosomes from synthetic chromosomal operons in Clostridium acetobutylicum ATCC 824. Biotechnol Biofuels. 2013;6(1):117.

39. Kubiak AM, Minton NP. The potential of clostridial spores as therapeutic delivery vehicles in tumour therapy. Res Microbiol. 2015;166(4):244-54.

40. Bao G, Wang R, Zhu Y, Dong H, Mao S, Zhang Y, Chen Z, Li Y, Ma Y. Complete genome sequence of Clostridium acetobutylicum DSM 1731, a solvent-producing strain with multireplicon genome architecture. J Bacteriol. 2011;193(18):5007-8.

41. Harris LM, Desai RP, Welker NE, Papoutsakis ET. Characterization of recombinant strains of the Clostridium acetobutylicum butyrate kinase inactivation mutant: need for new phenomenological models for solventogenesis and butanol inhibition? Biotechnol Bioeng. 2000;67(1):1-11.

42. Obrien RW, Morris JG. Oxygen and growth and metabolism of Clostridium-acetobutylicum. J Gen Microbiol. 1971;68:307-18.

43. Hartmanis MGN, Gatenbeck S. Intermediary metabolism in Clostridium acetobutylicum - levels of enzymes involved in the formation of acetate and butyrate. Appl Environ Microbiol. 1984;47(6):1277-83.

44. Sambrook J, Russell D. Molecular cloning: a laboratory manual. CSHL Press. 2001.

45. Horton RM, Hunt HD, Ho SN, Pullen JK, Pease LR. Engineering hybrid genes without the use of restriction enzymes: gene splicing by overlap extension. Gene. 1989;77(1):61-8. 